\title{
Lowering Operations of Large Steel Structures: Numerical Simulation \& Guidelines for a Safe Procedure
}

\author{
D. Lepikson Oliveira, PhD ${ }^{a}$, F. Rubin ${ }^{a}$, M.B. Pinheiro ${ }^{a}$, D.C. Braga ${ }^{a}$, L.F.S. Macedo ${ }^{b}$, \\ R.S. Macedo ${ }^{\text {b }}$, N. Szilard Galgoul, Dr.Ing. ${ }^{c}$, A.R. Sarabi, MSc. ${ }^{c}$ \\ aOODEBRECHT Engenharia \& Construção Internacional - Engenharia Industrial, R. Lemos Monteiro 120, \\ 12oandar, CEP 05501-050 - Butantã, São Paulo-SP,Brazil. \\ ${ }^{\text {b} E M A S A ~ E n g e n h a r i a ~ L t d a . ~ R . ~ P e t i t ~ C a r n e i r o ~ 1122, ~ c j . ~ 501, ~ C E P ~ 80240-130 ~-~ A ́ g u a ~ V e r d e, ~ C u r i t i b a-P R, B r a z i l . ~}$ \\ cNSG Engenharia, Projetos e Representação Comercial Ltda. R. Visconde de Inhaúma 134, Grupo 405, CEP \\ 20091-901 - Centro, Rio de Janeiro-RJ,Brazil.
}

\begin{abstract}
Lowering operations (or removal of temporary propping) are usually required as part of the assembling process of large steel structures under construction, in order to release them from the temporary supports, which represents the final step of the erection phase. This paper is dedicated to the study of this delicate engineering operation, covering two distinct approaches for the solution of the problem and addressing both the design and the execution phases. Indeed, two simple numerical models are proposed herein for the required structural analyses, so as to allow the check of compliance of the structure with the safety requirements prescribed by applicable standards for this transient phase, both for the structure itself, as well as for the temporary supports (in most cases, steel towers are used for this purpose). Additionally, an itemized procedure, aiming the achievement of a safe operation at the construction site, is also outlined. Two examples of successful operations, for which the proposed techniques were applied, are finally presented.
\end{abstract}

\section{INTRODUCTION}

The removal of temporary supports used during the erection phase of large steel structures, referred to in this article as a lowering operation, represents a big challenge for a structural engineer in charge, not necessarily because of the complexity of the assembly process itself, but due to the risk involved in the operation and the possible consequences of a potential failure.

This is a recurrent step to be overcome during the assembly process of a steel structure, whose erection sequence presumes the partition of the structure in smaller and simpler segments, simply supported by temporary work during the construction phase. Such segments (or modules) are preassembled at ground level, lifted up, held in their final pre-cambered position by temporary supports and subsequently connected to each other, until the complete structure is achieved and becomes selfsupporting. When this stage is reached, the temporary supports are no longer required and can all be removed. The operation of temporary support removal is referred to here as a lowering operation and is the main theme to be addressed in this article.

In Brazil, where the steel construction is yet incipient, this methodology was particularly adopted in the assembly of large roofs of football stadia, which were designed and built for the 2014 Football World Cup. One of these stadia is illustrated in Figure 1, the Corinthians Arena, in São Paulo, designed by the German office WERNER SOBEK (from Stuttgart) and constructed by the Brazilian company ODEBRECHT.

One should note in the example shown in Figure 1,that the temporary support members are usually constituted by steel towers, either prefabricated or tailor made structures specifically designed for such purpose ${ }^{1}$.

\footnotetext{
${ }^{1}$ In the particular case of the Corinthians Arena, these towers were an assembly of prefabricated members, constituted by modular components of a crane tower.
} 


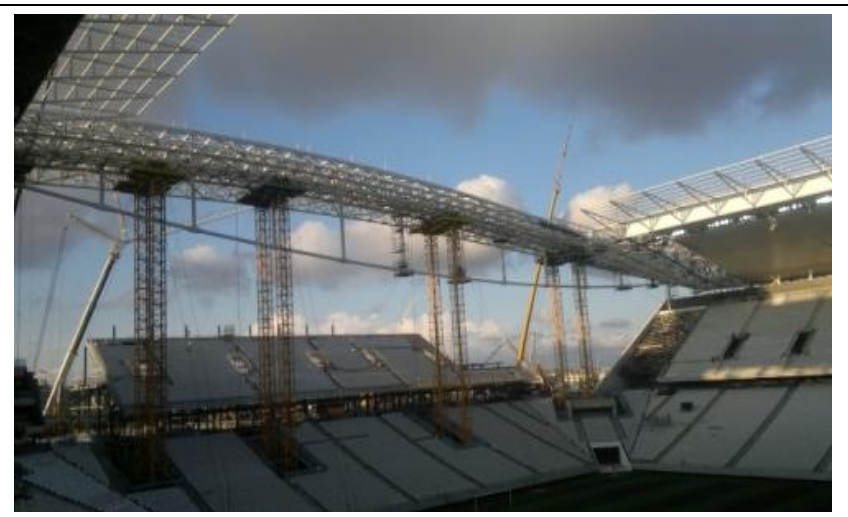

Figure1. Erection of the roof of the Corinthians Arena, in São Paulo, Brazil

But what are exactly the potential risks to which the engineers are subjected when such a construction procedure is carried out? The most important one - which actually determines the main variable to be controlled during the operation - lies in the possibility of an unexpected concentrated overload taking place at one of the temporary supports, leading to an increase of the corresponding reaction forces, which may be beyond the overall capacity of the tower (or the strength of some of its components). If such an event occurs, the overload will probably damage the new structure to be assembled and the overloaded support tower may even collapse, with severe consequences for the safety of the operation, aside from submitting the structure to an unpredicted dynamic load.

One should also notice that the main structure must always be checked for this temporary stage, in order to assure safety. Indeed, during the lowering process, the structure is submitted to particular loading and support conditions, so that part of its members may be subjected to internal forces that are very different from those predicted by the design and corresponding to the final stage (i.e. the complete structure, released from the temporary supports), either in intensity or nature (e.g. where tension forces are expected, compression forces may arise!).

At this point, it is already possible to highlight the following statements, revealed by the above introductory discussion:

- The basic variable to be controlled during the lowering operation is the magnitude of the reaction forces acting on the temporary supports, which are measured / imposed by suitable, previously selected hydraulic jacks, usually located on the top of the towers. These are the devices required in order to allow the step-by-step control of the displacements to be progressively imposed on the new assembled structure;

- The displacement of the structure is a secondary variable in the process, which is measured stepwise afterwards (i.e. after each relief on the controlled reactions), regarding which one has no control (since it is already intrinsically pre-determined by the overall behavior of the main structure, at the final stage, when it becomes totally free from the temporary supports);

- Both the temporary towers and the main structure must be checked for the construction phase, which comprises, in this case, the lowering operation, in order to determine whether the applied assembly procedure is safe or not. During this temporary phase, the structure is submitted to its dead weight, eventual construction loads and the applicable short-term wind (noting also that the structure may be partially assembled).

The main goal of this article is twofold as stated below:

- To propose a simple methodology to perform the numerical simulation of a typical steel structure lowering operation;

- To present a set of guidelines as a valuable aid for the preparation of a safe procedure to be implemented in the field.

The article is organized as follows:

- In Section2, an introductory explanation about the lowering operation is presented, based on the numerical simulation of a very simple structure during execution: a simply-supported concrete beam, temporarily supported by intermediate steel props during the erection phase; 
- In Section4, a simple (linear) numerical method to simulate a typical lowering operation is proposed, based on the linear superposition of a set of basic unit load results, which is referred herein as the Influence Matrix of the Reaction Force Method;

- In Section5, a set of guidelines is presented which should be observed in the preparation of a stepby-step procedure to be adopted in the field, as a preparation step for the execution of the lowering operations. These guidelines are based on previous experience accrued from similar operations;

- In Section6, some selected results obtained in former operations are presented, to illustrate the concepts discussed here. They correspond to the construction phase of the roof of two stadia: the Corinthians Arena (Football World Cup 2014), in São Paulo; and João Havelange Olympic Stadium (Olympic Games 2016), in Rio de Janeiro;

- Finally, in Section7, some relevant comments and final remarks are issued.

The references indicated throughout the text are presented at the end of this document.

\section{Conceptual Model}

Consider the theoretical structure illustrated in Figure2: a statically-determined beam with a $30 \mathrm{~m}$ span, supported by four props along the span in its initial configuration.

The main idea to be exploited in this simple example is to show how to migrate from a continuous system to a simply-supported beam, by progressively imposing proper intermediate displacements, aiming to achieve the final desired configuration. The stepwise displacements are controlled by a suitable relief in the intermediate reaction forces, using hydraulic devices for that purpose.

In this case, one may wonder how the temporary reactions should vary, in order to obtain a deflected shape, which remains proportional to the final configuration at each step -- something to be predicted by the procedure to be adopted in the field. Practical issues similar to this one may arise and are addressed in this example.

Initial structural system:

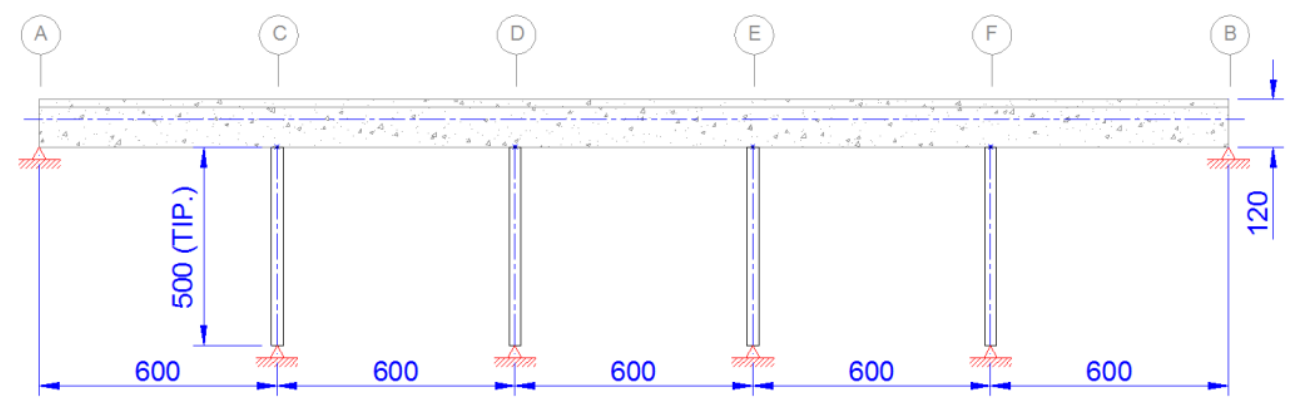

Final structural system:

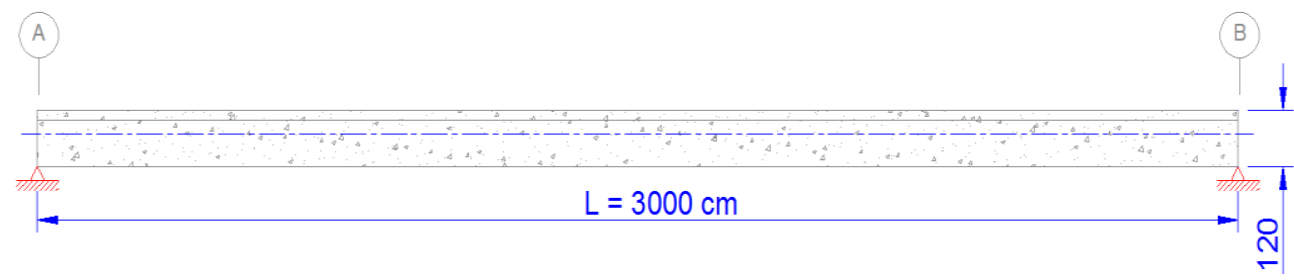

Cross sections:

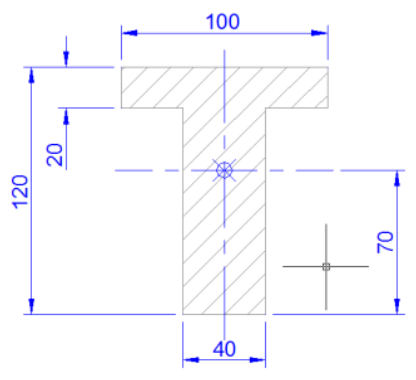

Concrete beam

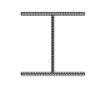

HP $310 \times 79$

Steel props

Figure2. A simple case of a lowering operation: a simple concrete beam propped during the erection phase. 


\begin{tabular}{|c|c|}
\hline$A$ & ............Cross-sectional area; \\
\hline$L$ & 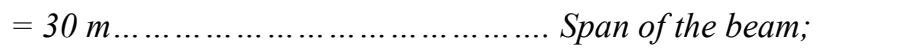 \\
\hline$\gamma_{c}$ & ....Density of concrete; \\
\hline$W$ & .. Total weight of the beam; \\
\hline$w$ & ... Distributed dead load. \\
\hline
\end{tabular}

The main ideas of the numerical method applied here are summarized below.

\subsection{Purpose}

To determine the reaction forces acting in each step on the temporary/intermediate supports, starting from the initial configuration (a continuous beam) up to the final configuration (a simply-supported beam), considering that the operation is performed in " $n$ " steps and that the deflections of the beam for the final configuration are known. ${ }^{2}$

\subsection{Method}

To calculate the loading mobilized step-by-step by the structural system due to a relief of the previously determined support reactions, allowing the structure to displace stepwise at the temporarily supported intermediate points, using the following equation (written for a generic Step $i$ ):

$[\Delta F]_{m \times 1}^{i}=[K]_{m \times m}[\Delta \mathrm{U}]_{m \times 1}^{i}$

The Stiffness Matrix $[K]$ is obtained from the Flexibility Matrix $[L]$, as follows:

$[K]_{m \times m}=[L]_{m \times m}^{-1}$,

Whereas the matrix $[L]$ is easily determined for a simple / statically determined system (in this case, a simply-supported beam). Indeed,

Column $j$ of matrix $[L]=L_{i j}, i=1 \ldots m=$ Displacements of the beam at the points $i=1 \ldots m$ due to a unit load P, applied at position $j$ along the beam, $j=1 \ldots m$-See Figure3.

Conversely,

Column $j$ of matrix $[K]=K_{i j}, i=1 \ldots m=$ Set of forces acting on the beam at the points $i=1 \ldots m$ that cause a unit displacement at position $j$ along the span, $j=1 \ldots m$.

\subsection{Main Assumptions}

- The supports are considered rigid, for simplification purposes, and were replaced by the corresponding (initial) reaction forces, acting upon a statically determined structure;

- The system is linear (i.e. submitted to small displacements and strains), so that the solution in each step may be obtained by superposition, starting from the initial structure (at Step 0), which corresponds, in this case, to a continuous beam.

The notation used herein is summarized below:

\begin{tabular}{|l|l|l|}
\hline$[K]_{m \times m}$ & $=$ & Stiffness matrix; \\
\hline$[L]_{m \times m}$ & $=$ & Flexibility matrix; \\
\hline$[\Delta F]_{m \times 1}^{i}$ & $=$ & $\begin{array}{l}\text { Variation on the imposed Reaction Forces acting on the supports, at Step } \\
i ;\end{array}$ \\
\hline$[\Delta \mathrm{U}]_{m \times 1}^{i}$ & $=$ & Displacements at the intermediate support points, at Step $i ;$ \\
\hline$m$ & $=$ & Number of intermediate supports; \\
\hline$n$ & $=$ & Number of steps (Procedure). \\
\hline
\end{tabular}

\footnotetext{
${ }^{2}$ This is always available, since it is standard information determined by the designer: the deformed shape of the structure when subjected to its self-weight.
} 


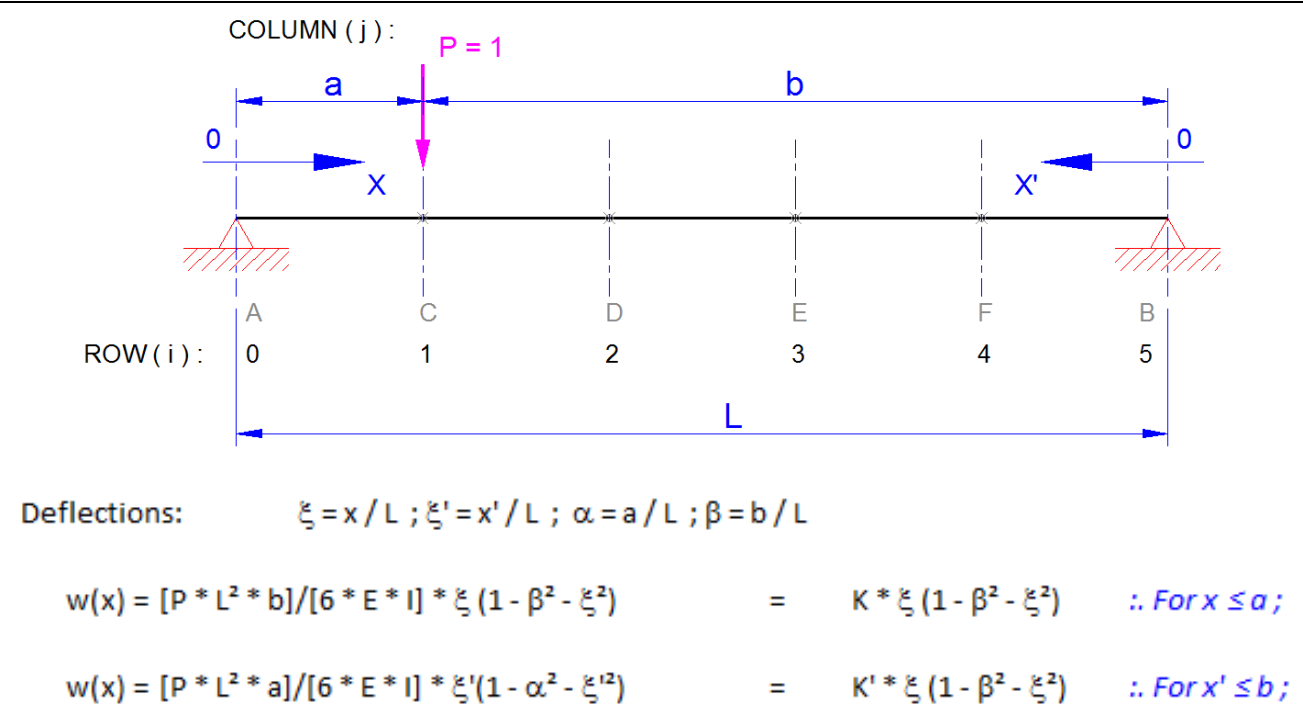

Figure3. Scheme for the calculation of the Flexibility Matrix $[\boldsymbol{L}]_{m \times m}$.

\section{RESULTS}

Applying the proposed procedure in the numerical example presented in Figure2, one obtains the following results:

\subsection{Reaction Forces}

For the INITIAL SYSTEM (Continuous beam):

$\mathrm{R}_{\mathrm{A}}=\mathrm{R}_{\mathrm{B}}=3.56 \mathrm{tf}$

$\mathrm{R}_{\mathrm{C}}=\mathrm{R}_{\mathrm{F}}=10.19$ tf

$\mathrm{R}_{\mathrm{D}}=\mathrm{R}_{\mathrm{E}}=8.77 \mathrm{tf}$

FINAL SYSTEM (Simply-supported beam):

$\mathrm{R}_{\mathrm{A}}=\mathrm{R}_{\mathrm{B}}=22.50 \mathrm{tf}$

$\mathrm{R}_{\mathrm{C}}=\mathrm{R}_{\mathrm{D}}=\mathrm{R}_{\mathrm{E}}=\mathrm{R}_{\mathrm{F}}=0$

\subsection{Displacements (final configuration)}

Table1. Displacements at support points along the beam, final stage.

\begin{tabular}{rrrrrr}
\hline Support & $\begin{array}{r}\mathrm{x} \\
{[\mathrm{cm}]}\end{array}$ & $\mathrm{x} / \mathrm{L}$ & $\begin{array}{r}\mathrm{f}(\xi) \\
{[\mathrm{cm}]}\end{array}$ & $\begin{array}{r}\Delta \\
{[\mathrm{cm}]}\end{array}$ & $\mathrm{L} / \Delta$ \\
\hline$A$ & 0 & 0.00 & $\mathbf{0 . 0 0 0}$ & $\mathbf{0 . 0 0}$ & - \\
$C$ & 600 & 0.20 & $\mathbf{0 . 1 8 6}$ & $\mathbf{4 . 4 0}$ & $\mathbf{6 8 3}$ \\
$D$ & 1200 & 0.40 & $\mathbf{0 . 2 9 8}$ & $\mathbf{7 . 0 5}$ & $\mathbf{4 2 6}$ \\
$E$ & 1800 & 0.60 & $\mathbf{0 . 2 9 8}$ & $\mathbf{7 . 0 5}$ & $\mathbf{4 2 6}$ \\
$F$ & 2400 & 0.80 & $\mathbf{0 . 1 8 6}$ & $\mathbf{4 . 4 0}$ & $\mathbf{6 8 3}$ \\
$\mathrm{B}$ & 3000 & 1.00 & $\mathbf{0 . 0 0 0}$ & $\mathbf{0 . 0 0}$ & - \\
$\mathrm{w}(\mathrm{L} / 2)=\mathbf{7 . 4 0} \mathrm{cm}$ & $=\mathrm{L} / \mathbf{4 0 5 .}$ & & & &
\end{tabular}

\subsection{Structural Matrices}

$m=4$ intermediate supports;

Flexibility Matrix $[L]$, in $[\mathrm{cm} / \mathrm{tf}]$ : 


\begin{tabular}{|c|c|c|c|c|c|}
\hline & & 1 & 2 & 3 & 4 \\
\hline \multirow{4}{*}[\mathrm{L}]{$=$} & 1 & 0.107771 & 0.151553 & 0.134713 & 0.077460 \\
\hline & 2 & 0.151553 & 0.242484 & 0.229013 & 0.134713 \\
\hline & 3 & 0.134713 & 0.229013 & 0.242484 & 0.151553 \\
\hline & 4 & 0.077460 & 0.134713 & 0.151553 & 0.107771 \\
\hline
\end{tabular}

Stiffness Matrix $[K]$, in $[t f / c m]$ :

\begin{tabular}{|c|c|c|c|c|c|}
\hline & & 1 & 2 & 3 & 4 \\
\hline \multirow{4}{*}[K]{$=$} & 1 & 97.744 & -94.050 & 40.916 & -10.229 \\
\hline & 2 & -94.050 & 138.660 & -104.279 & 40.916 \\
\hline & 3 & 40.916 & -104.279 & 138.660 & -94.050 \\
\hline & 4 & -10.229 & 40.916 & -94.050 & 97.744 \\
\hline
\end{tabular}

\subsection{Lowering Operation / Results}

$n=5$ steps;

Displacements, in $[\mathrm{cm}]$ :

The discrete displacements $[\Delta \mathrm{U}]_{m \times 1}^{i}$ are predefined, in order to obtain equally spaced increments on each intermediate support, as shown in Table2.

Table2. Displacements at support points along the beam, in each step.

\begin{tabular}{ccccrrrr}
\hline STEP & 0 & \multicolumn{7}{c}{1} & 2 & 3 & 4 & 5 \\
\hline SUpport & & \multicolumn{7}{c}{ DISPLACEMENTS, $\mathrm{cm}$} \\
\hline$A$ & 0 & 0.00 & 0.00 & 0.00 & 0.00 & 0.00 & $\mathbf{0 . 0 0}$ \\
$C$ & 1 & 0.00 & -0.88 & -1.76 & -2.64 & -3.52 & $-\mathbf{4 . 4 0}$ \\
$D$ & 2 & 0.00 & -1.41 & -2.82 & -4.23 & -5.64 & $-\mathbf{7 . 0 5}$ \\
$E$ & 3 & 0.00 & -1.41 & -2.82 & -4.23 & -5.64 & $-\mathbf{7 . 0 5}$ \\
$F$ & 4 & 0.00 & -0.88 & -1.76 & -2.64 & -3.52 & $-\mathbf{4 . 4 0}$ \\
$B$ & 5 & 0.00 & 0.00 & 0.00 & 0.00 & 0.00 & $\mathbf{0 . 0 0}$ \\
\hline
\end{tabular}

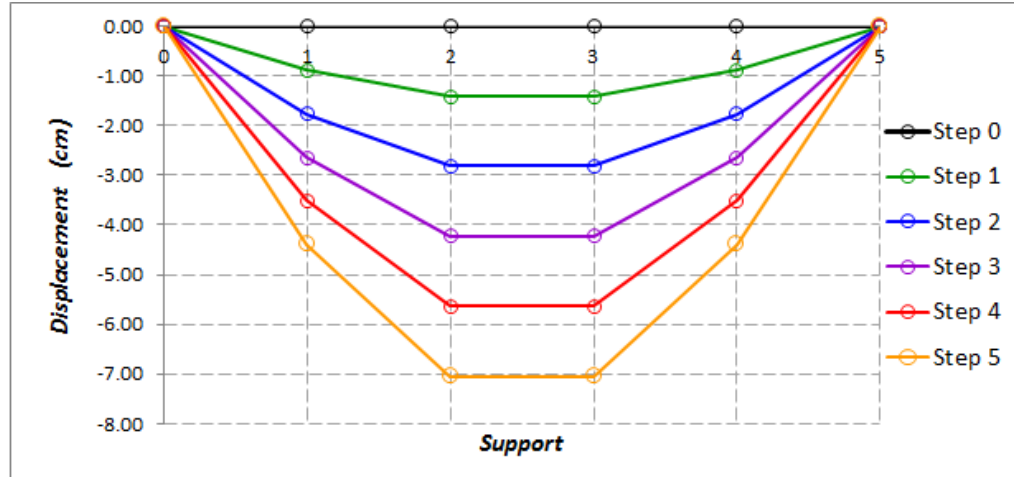

Reaction forces, in $[t f]$ :

Table3.Reaction forces at the support points, in each step.

\begin{tabular}{crrrrrrr}
\hline STEP & & 0 & \multicolumn{7}{c}{1} & 2 & 3 & 4 & 5 \\
\hline SUpport & \multicolumn{7}{c}{ REACTION FORCES, $t f$} \\
\hline$A$ & 0 & 3.55 & 7.34 & 11.12 & 14.91 & 18.70 & $\mathbf{2 2 . 4 9}$ \\
$C$ & 1 & 10.19 & 8.15 & 6.11 & 4.08 & 2.04 & $\mathbf{0 . 0 0}$ \\
$D$ & 2 & 8.77 & 7.01 & 5.26 & 3.51 & 1.76 & $\mathbf{0 . 0 0}$ \\
$E$ & 3 & 8.77 & 7.01 & 5.26 & 3.51 & 1.76 & $\mathbf{0 . 0 0}$ \\
$F$ & 4 & 10.19 & 8.15 & 6.11 & 4.08 & 2.04 & $\mathbf{0 . 0 0}$ \\
$B$ & 5 & 3.55 & 7.34 & 11.12 & 14.91 & 18.70 & $\mathbf{2 2 . 4 9}$ \\
\hline
\end{tabular}




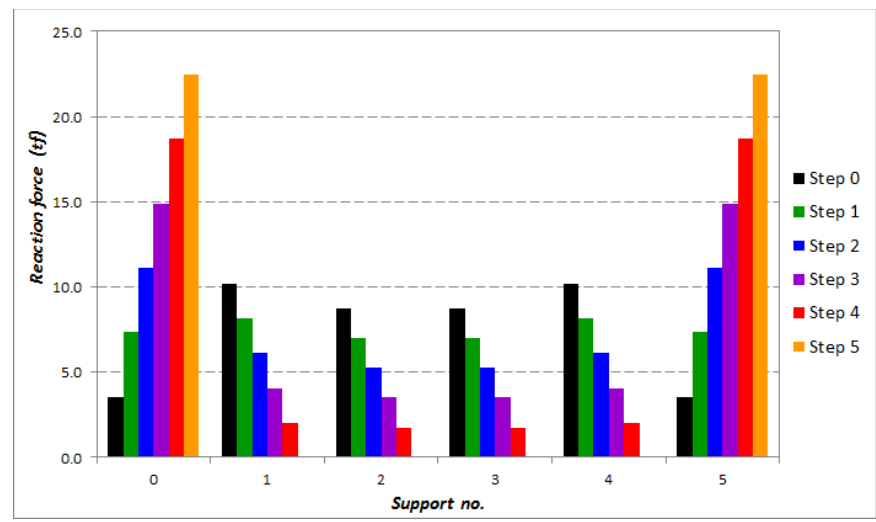

One should notice that the curves plotted to illustrate the data presented in Table 2 do not exactly represent the deformed shape of the beam, since the displacements were evaluated only at the support points. This is the information usually available in practice, obtained by a topographic survey, as part of the activities to monitor the operation, as discussed in the sequence (See Item 5).

\subsection{Relevant Commentaries and Remarks}

The study of such a simple and straightforward example reveals some important (and yet obvious) findings, summarized below. These remarks are also valid for more general and complex structures when submitted to a lowering operation to remove the temporary intermediate supports used during its erection phase:

- Either one of the variables, namely, displacements or reaction/hydraulic forces (both measured at the supported points), may be chosen as the main parameter to control the lowering operation, leading to exactly the same results. The choice of one or the other depends on practical issues. Since the magnitude of the reaction forces is an issue regarding the safety margin of the towers, and due to the fact that they are easily controlled by the hydraulics, the reaction forces are usually preferred for this purpose;

- It is already quite clear that the beginning of the process is the most risky phase, because the magnitude of the reaction forces are higher, i.e. close enough to their initial / maximum values (and they tend to reduce as the process progresses). During this initial phase, much care should be taken, in order to avoid unexpected migration and load distribution between the temporary supports. This is prone to occur when working with statically indeterminate structures, as is normally the case;

- For this "classical approach" just presented, in which the load proportionally varies on all supports throughout the whole lowering operation, the main structure is automatically checked for the erection phase, excluding the handling of the component modules, that require specific checks, since the first and the last phases correspond to the most critical stages, i.e. the Main Structure totally supported (on temporary jack supports) and the Final Configuration (simply supported beam), respectively. During the lowering operation, the main structure is forcibly submitted to a less severe loading, as one may observe in Figure4, for the example discussed here;

- Although this is a convenient outcome, it also has the disadvantage that all the temporary towers will be liberated for disassembly at the same time and only by the end of the construction works (this can be troublesome and may cause some inconvenience for the construction team regarding planning and deadlines...).

- A lowering operation may be regarded as just a safe way to ensure that the self-weight will be statically applied and progressively resisted by the newly-assembled structure, during which the reaction forces acting on the temporary towers are controlled, in order to avoid unexpected load distributions between temporary supports;

- The variation $[\Delta F]_{m \times 1}^{i}$ on the reaction forces represents the load share transferred to the structure at step $\boldsymbol{i}$, while the towers are relieved by the same amount. This is a cumulative process, until the structure becomes free from the temporary supports and resists its self-weight on its own (as shown in Table3). 
This approach to remove the temporary supports is referred to herein as the Method of Related Deformed Shapes.

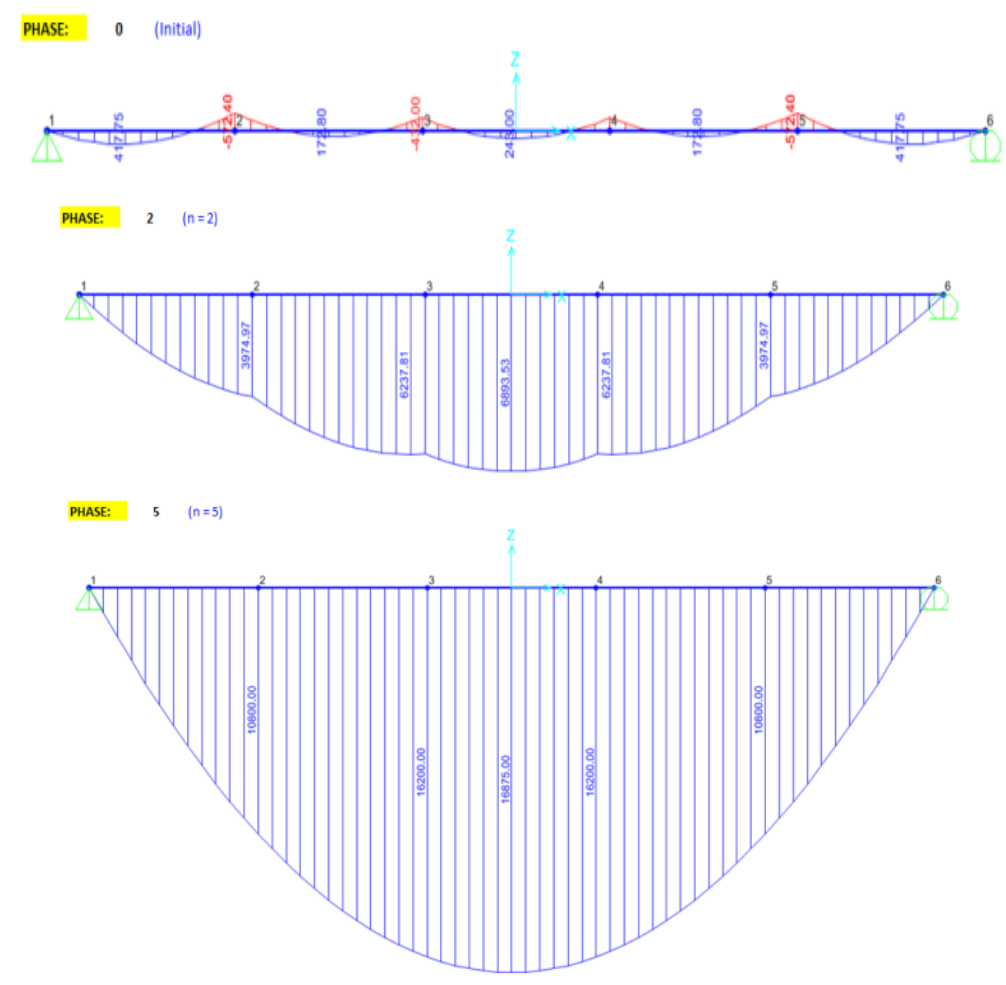

$\operatorname{Mmax}=16875 \mathrm{tf}^{*} \mathrm{~cm}$

Figure4. Internal forces (Bending Moment, in $t f^{*} \mathrm{~cm}$ ) acting on the beam during the erection sequence (for $n=$ 0,2 and 5).

The method presented above, based on the use of a flexibility matrix, was a very useful tool to address some basic and important ideas about the topic discussed in this article. Some relevant and generally applicable conclusions came up based on the solution of a simple example, as discussed above in Paragraph (3.5).Although still applicable, this method is not convenient to solve real and more complex problems, because it would require the aid of a computer to perform the calculations anyway. For this purpose, another numerical method to simulate a lowering operation is presented in the next section (Item 4), which is still simple, but much more efficient if compared to the one just presented.

\section{Proposed Numerical Model to Simulate the Lowering Operation}

Now that the main concepts were introduced, it is desirable to generalize the idea just presented and develop a systematic numerical procedure, which is also capable to solve real (and consequently, more complex) structures in a practical way. A suitable method for this purpose is now presented. It was conceived in such a way that no sophisticated finite-element based software is required. Indeed, a finite element tool, commonly available in design offices, may be used for such purpose (or any other software based on simpler matrix methods, depending, of course, on the type of structure to be analyzed) to perform a conventional linear analysis.

The main differences of the general method now proposed, if compared to the one previously presented, are pointed out below:

- The temporary supports are removed one at a time, following a predefined sequence, and no longer all at the same time during the last step of the lowering operation;

- The stiffness of the support towers is now taken into account and determines the loading distribution after a certain support is removed from the system. 
As a consequence, it is no longer automatically assured that the structure can withstand the applied loading during the lowering operation, as occurred in the previous case discussed in Item 2, referred to herein as the "classical approach". Instead, the safety of each structural member must be checked beforehand. These verifications lead to a suitable sequence to be chosen, so that the progressive removal of the temporary towers is a safe procedure, if possible. If not, the classical approach must be employed instead. It must be clear, therefore, that this is a trial-and-error procedure and that this extra work cannot be avoided.

This alternative approach is referred to in this article as the Influence Matrix method for the Reaction Forces, which is summarized in the sequence.

It is already clear that the proposed alternative method is more time consuming, if compared to the classical approach previously presented. However, one must consider that all the numerical calculations are fast and easily made with the aid of a personal computer, performing conventional structural analysis.

The main advantage that justifies the choice of this more complex procedure is actually operational, in the construction site, due to the possibility to disassemble the temporary towers earlier, as they are being released, with the corresponding saving of time, cost and Work Planning of the enterprise.

After this brief introduction, the Influence Matrix method for the Reaction Forces is now presented.

\subsection{Purpose}

To establish a general procedure for the numerical simulation of a lowering operation, in which the temporary towers may be progressively removed (i.e. the structural system continuously changes), in order to:

- Define a proper sequence to remove the towers (one at a time);

- Calculate the reaction forces accordingly, taking into account the stiffness of the temporary towers in the loading distribution between the intermediate supports during the operation;

- Check the main structure during the operation, to ensure that each one of its structural members is capable to withstand the construction phase safely.

Typically, the lowering operation comprises all the intermediate steps from a continuously supported structure up to the final configuration, corresponding to the assembled structure supported at both ends and submitted to its self-weight.

\subsection{Method}

The following data are known beforehand:

- The Initial reaction forces, corresponding to the initial configuration of the structural system = Main structure totally assembled and resting upon the temporary supports;

- The final configuration of the structural system = Main structure in the final position and submitted to dead load, comprising its deformed shape (for comparison purposes during the execution phase).

\subsection{Main Assumptions}

Once again, it is assumed that the operation is executed in " $n$ " steps and that the deflections of the structure for the final configuration are known, as usual.

It is also assumed that a Finite Element-based software is available to automatically perform the required structural calculations. One should note that the nonlinearities involved are limited to problem of determining the load distribution after the removal of a support, via the Influence Matrix method, as detailed below (i.e. the structural system changes after each step). Using this approach, a non-linear "Staged Construction" type analysis is avoided (although it would be indeed a straightforward alternative for the same purposes pursued here). 


\section{Lepikson Oliveira et al.}

The numerical procedure was developed with the basic assumption that one tower is removed per step, up to the total release of the structure from the temporary supports.

It is also assumed that the structure and the temporary towers were checked for the initial configuration and that strengthening, if required, was already installed and computed as part of the applied dead load.

The final configuration is never an issue, since the dead load is only part of the loading, to which the structure will be subjected during its use (and, consequently the structure was designed to resist it).

The proposed method is summarized in the following steps:

1. Define tentatively a suitable sequence to progressively remove the temporary towers (one at a time);

2. For the sequence chosen, proceed with the calculation of the Influence matrix for the reaction forces, i.e. the loading distribution on the remaining supports when a unity load is applied at the position of the tower to be removed. There will be as many sets of "unit" (or "normalized") reaction forces as the number of steps, considering that one tower is removed in every step; Notice that each set of reaction forces represents a line of the Influence matrix, with a zero element placed in the position corresponding to the tower to be removed.

3. Compute the loading variation on the remaining supports, for each step. These increments are easily obtained from the influence matrix, multiplying the "unit" reactions by the magnitude of the actual load (the one which corresponds to the reaction force acting on the tower to be removed in the given step;

4. Compute the sum of the applied forces on the remaining towers, by just adding the increments in each step to the initial reaction;

5. To determine the behavior of the structure in each phase or step " $i$ " $(i=1 \ldots n)$, one must work with the structural model in the final configuration (i.e. supported at the ends and completely free from the intermediate supports) and apply the following loading, composed by:

- The initial loading (including the reaction forces at the intermediate supports);

- The final reaction at the position corresponding to the tower that was removed in step $i$, but applied in the direction of gravity (i.e. loading the structure);

- The final reactions at the positions where the towers were already removed, in the previous steps $(i-1, i-2, \ldots)$, are also applied in gravity direction;

- The accumulated increments at the remaining positions, where the other towers are located and are still loaded, supporting the main structure.

6. Check both the structure and the towers in each step " $\mathrm{i}$ " (i=1 ...n-1), in order to ensure that the structural members can safely withstand the applied loading, as per applicable standards.

Following the procedure presented above up to the end, after step " $n$ " (i.e. after the removal of the last tower), it is evident that the structure will become submitted to the initial loading, but without the intermediate supports, or in its final configuration. This outcome, of course, does not depend on the sequence chosen for the removal of the towers.

To justify this method -which is based on the linear superposition of the results corresponding to the loading distribution on each support- it suffices to recognize that the structure "feels" only the loading increments due to the stepwise removal of the towers, noting that a complementary part of the reaction force remains on the towers, whenever they are active.

However, based on the outcome of STEP 6 above, it may be necessary to start the procedure over again, trying a different removal sequence of the towers, in order to obtain a better distribution of the temporary reaction forces on the intermediate supports; or of the internal forces acting on the partially supported structure.

The procedure to obtain the Influence Matrix for the reaction forces (STEP 2) is illustrated in Figure5, in this case for a simple beam temporarily supported by four intermediate spring supports: 

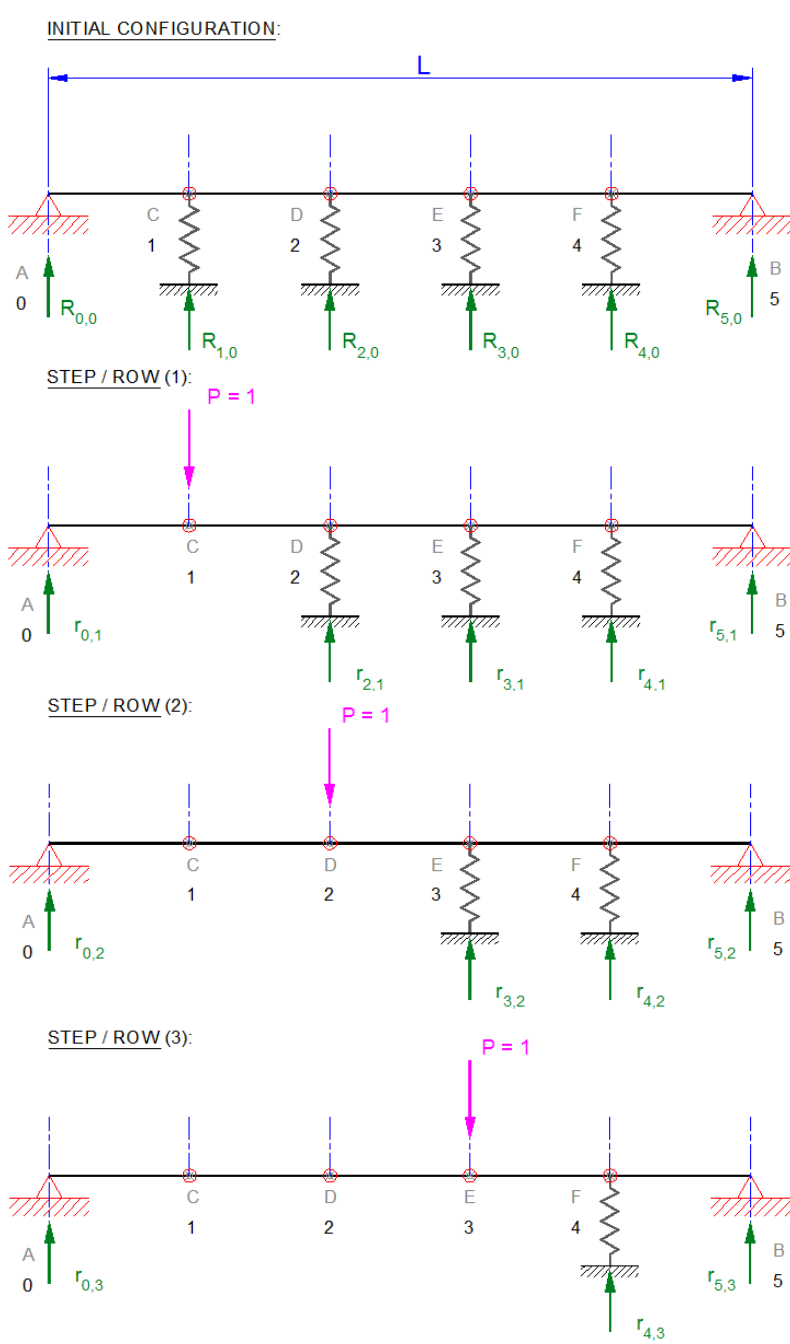

STEP $(4)=$ FINAL CONFIGURATION

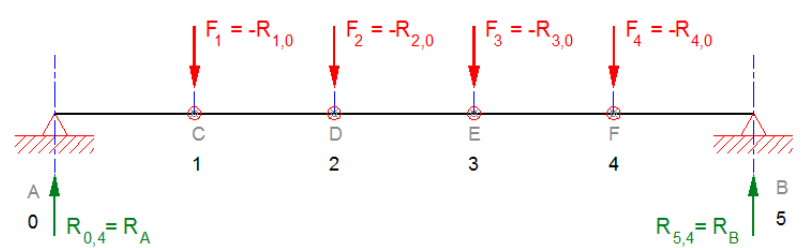

Figure5. Calculation scheme for the Influence Matrix of the reaction forces.

Notice that the supports are progressively removed as the unit load $P$ changes its position, as already emphasized. In this example, the sequence 1-2-3-4 was arbitrarily chosen for that purpose, but it could be any other (as long as the structure resists the corresponding internal forces) and not necessarily in a sequential order.

In Figure 5, the following notation was used:

\begin{tabular}{|l|l|l|}
\hline$R_{i, j}$ & $=$ & Reaction force at Support $[i]$, in $\operatorname{Step} j ;$ \\
\hline$P$ & $=$ & Unit Load $;$ \\
\hline$r_{i, j}$ & $=$ & $\begin{array}{l}\text { Reaction Force at Support }[i], \text { in Step } j, \text { due to the unit load } P \text { (an element of the Influence } \\
\text { Matrix); }\end{array}$ \\
\hline$F_{i}$ & $=$ & Force transferred to the structure after the removal of Support $[i]$. \\
\hline
\end{tabular}

At each step, the reaction force in any active Support [i] may be obtained by (See also Table6):

$R_{i, k}=R_{i, 0}+\sum_{j=1}^{k} \Delta R_{i, j}=R_{i, 0}+\sum_{j=1}^{k}\left(-R_{p, j-1}\right) \cdot r_{i, j}$ 
where:

\begin{tabular}{|l|l|l|}
\hline$i$ & $=$ & $1 \ldots m$, being $m$ the total number of intermediate supports; \\
\hline$k$ & $=$ & Step during which the Support Reaction $R_{i, k}$ is to be evaluated $(k<n) ;$ \\
\hline$\Delta R_{i, j}$ & $=$ & Accumulated Reaction Force at Support $[i]$, at Step $j ;$ \\
\hline$-R_{p, j}$ & $=$ & $\begin{array}{l}\text { Accumulated load acting on Support }[p] \text { up to the previous step }(i . e . \text { up to Step } j-1<k), \\
\text { applied on the structure in Step } j \text { due to the removal the corresponding tower, being } p \neq i \\
\text { (the negative sign is just to emphasize that the load is applied on the structure, in the } \\
\text { opposite direction). }\end{array}$ \\
\hline
\end{tabular}

The reaction forces acting on the temporary towers must obviously be monitored during the lowering operation, as it will be further discussed in Section 5.

\subsection{Numerical Example}

The most effective way to illustrate the proposed method is by presenting a simple numerical example: a two-dimensional statically-determined truss, temporarily supported by five intermediate elastic supports.

The problem is summarized in Figure6below:

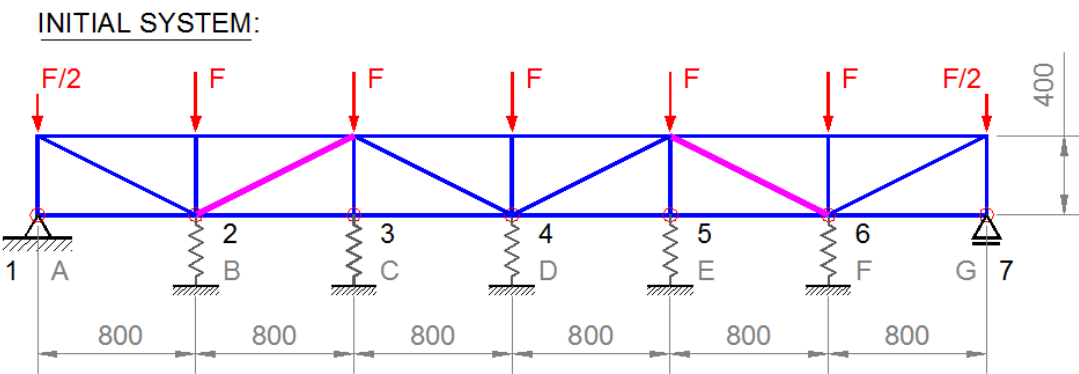

FINAL SYSTEM:

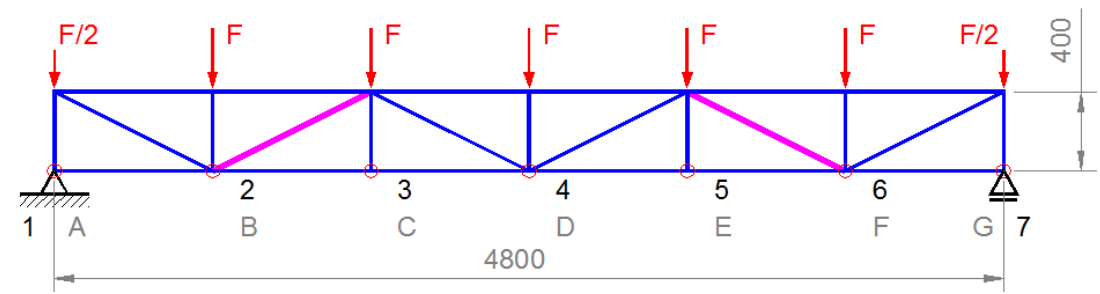

Figure6. Numerical example: Truss with intermediate elastic supports (dimensions in $\mathrm{cm}$ ).

\subsubsection{Geometrical properties (American Profiles)}

\begin{tabular}{|l|l|}
\hline Upper Chord $=$ & Profile W $610 \times 155$ \\
\hline Lower Chord $=$ & Profile W 200 $\times 46.1$ \\
\hline Vertical members $=$ & Profile W 250 $\times 44.8$ \\
\hline Diagonals $=$ & Profile W $360 \times 57.8$ (Typical) \\
\hline & Profile W $310 \times 79.0$ (Highlighted) \\
\hline
\end{tabular}

\subsubsection{Elastic properties of the supports}

Vertical stiffness $\left(k_{z}\right)=101.97 \mathrm{tf} / \mathrm{cm}(1000 \mathrm{kN} / \mathrm{cm})$

All the elastic supports have the same stiffness $k_{z}$, which corresponds to the axial stiffness of the temporary towers.

4.4.3. Material properties: Structural steel

$\gamma=7.85 \mathrm{tf} / \mathrm{m}^{3}\left(77.0 \mathrm{kN} / \mathrm{m}^{3}\right)$ Weight Density

$E=2.05 \times 10^{3} \mathrm{tf} / \mathrm{cm}^{2}\left(20.11 \times 10^{3} \mathrm{kN} / \mathrm{cm}^{2}\right) \ldots$. Young's modulus

$v=0.30$. Poisson's ratio

\subsubsection{Loading}

The self-weight of the truss is disregarded in this example, for the sake of simplicity (and also to be faithful to the theoretical truss model). 
Lowering Operations of Large Steel Structures: Numerical Simulation \& Guidelines for a Safe Procedure

To simulate the complete lowering operation, the following Load Cases are to be considered:

\begin{tabular}{|l|l|l|}
\hline LC.01 & $=$ & $\begin{array}{l}\text { External loads F = 8.154 tf }(80 \mathrm{kN}), \text { applied at the nodes of the upper } \\
\text { chord of the truss; }\end{array}$ \\
\hline LC.02 to 06 & $=$ & $\begin{array}{l}\text { Reaction forces corresponding to each support, according to the } \\
\text { sequence of removal chosen; }\end{array}$ \\
\hline LC.07 to 10 & $\begin{array}{l}\text { Set of loads to simulate the distribution of forces between the remaining } \\
\text { support points, due to the progressive removal of the towers, } \\
\text { corresponding STEP 01 ( = LC.07, for the first tower) to STEP 04 ( } \\
\text { LC.10, for the penultimate tower). }\end{array}$ \\
\hline LC.11 to 15 & $=$ & $\begin{array}{l}\text { Accumulated load to be applied on the structure due to the release of a } \\
\text { given tower, according to the sequence of removal chosen (LC.11 = First } \\
\text { tower, ..., LC.15 = Fifth/last tower). }\end{array}$ \\
\hline
\end{tabular}

The operation is simulated by suitable superposition of the above described load cases, as will be demonstrated in the sequence, so that the structural system evolves from a multi-supported structure to the final (statically-determined) configuration.

Therefore, the sequence to be chosen for the removal of the towers clearly impacts the loading to be applied, as well as the determination of the influence matrix itself (since the structural system changes!). So, if a new sequence is to be studied, the numerical model shall be redone from the very beginning.

\subsubsection{Analysis assumptions}

End releases were set for all structural members, to simulate pin-connections of an ideal truss model.

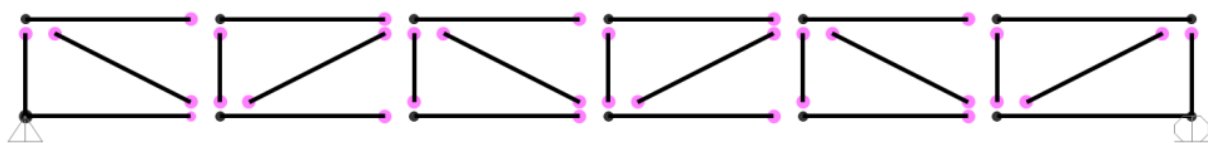

The commercial software SAP2000 (Ref. [1])was chosen to solve the structural model presented here.

The safety of the structure is checked as per AISC / ASD criteria (Ref. [2]), which is based on the Working Stress Approach, just to render the code check simpler and straightforward, since this is not the main purpose of the study presented here.

The supports were simulated as simple elastic springs and are not checked in this numerical example (even though this is one of the most important checks to be performed in a real case, to ensure the integrity of the towers, especially in the beginning of the operation, when the reaction forces are larger).

\subsubsection{Numerical Solution}

The solution scheme presented below follows the same steps as previously outlined in the presentation of the proposed method.

1. Sequence chosen to remove the towers (See Figure6):

Supp. $02 \rightarrow$ Supp. $06 \rightarrow$ Supp. $03 \rightarrow$ Supp. $05 \rightarrow$ Supp. 04

2. Assembling of the Influence Matrix for the Reaction Forces:

Table4. The influence matrix for the Reaction Forces.

\begin{tabular}{cccccr}
\hline STEP & SUPP.02 & SUPP.03 & SUPP.04 & SUPP.05 & SUPP.06 \\
\hline \multicolumn{7}{c}{ Initial Reaction Forces $\mathrm{R}_{\mathrm{i}, 0}(\mathrm{kN})$} \\
\hline $0=$ Begin & $\mathbf{7 3 . 6 8 6}$ & $\mathbf{7 5 . 9 5 5}$ & $\mathbf{8 9 . 0 7 7}$ & $\mathbf{7 5 . 9 5 5}$ & $\mathbf{7 3 . 6 8 6}$ \\
\hline \multicolumn{6}{c}{ Unitary Reaction Force $\mathrm{r}_{\mathrm{i}, \mathrm{j}}(\mathrm{kN})$, for $\mathrm{P}=1.0 \mathrm{kN}$} \\
\hline 1 & $\mathbf{0}$ & 0.44710 & 0.09296 & -0.01343 & -0.01120 \\
\hline 2 & - & -0.01844 & 0.09193 & 0.44731 & $\mathbf{0}$ \\
\hline 3 & - & $\mathbf{0}$ & 0.82560 & -0.00746 & - \\
\hline 4 & - & - & 0.81949 & 0 & - \\
\hline $\mathrm{r}_{\mathrm{i}, \mathrm{j}}=0$ & :. Application point of the Unit Load $\mathrm{P}=1.0 \mathrm{kN}$ \\
\hline
\end{tabular}


D. Lepikson Oliveira et al.

3. Load increments of the Reaction Forces:

Table5. Load Increments of the Reaction Forces, per step.

\begin{tabular}{|c|c|c|c|c|c|}
\hline STEP & SUPP.02 & SUPP.03 & SUPP.04 & SUPP.05 & SUPP.0 \\
\hline \multicolumn{6}{|c|}{ Load increments $\Delta \mathrm{R}_{\mathrm{i}, \mathrm{j}}$ in each STEP $(k N)$} \\
\hline 1 & 0 & 32.945 & 6.850 & -0.990 & -0.82 \\
\hline 2 & - & -1.344 & 6.698 & 32.591 & \\
\hline 3 & - & 0 & 88.799 & -0.802 & \\
\hline 4 & - & - & 87.484 & 0 & \\
\hline \multicolumn{6}{|c|}{$\Delta \mathrm{R}_{\mathrm{i}, \mathrm{j}}=\mathrm{r}_{\mathrm{i}, \mathrm{j}}{ }^{*}$ App. Load (i) } \\
\hline & Infl. Matrix & \multirow{6}{*}{\multicolumn{4}{|c|}{$\begin{array}{l}\text { To calculate the load increments: } \\
\Delta R_{i, j}=r_{i, j} * \text { App. Load }(j) ; \\
\Delta R_{i, j}=0 \rightarrow \text { Support is removed. }\end{array}$}} \\
\hline STEP & *App. Load (kN) & & & & \\
\hline 1 & 73.686 & & & & \\
\hline 2 & 72.861 & & & & \\
\hline 3 & 107.556 & & & & \\
\hline 4 & 106.754 & & & & \\
\hline
\end{tabular}

4. Accumulated Reaction Forces, per step:

Table6. Accumulated Reaction Forces, per step.

$\begin{array}{llllll}\text { STEP } & \text { SUPP.02 } & \text { SUPP.03 } & \text { SUPP.04 } & \text { SUPP.05 } & \text { SUPP.06 }\end{array}$

\begin{tabular}{|c|c|c|c|c|c|}
\hline \multicolumn{6}{|c|}{ Accumulated Reaction $\mathrm{R}_{\mathrm{i}, \mathrm{j}}$ in each STEP $(k N)$} \\
\hline 1 & 0 & 108.900 & 95.927 & 74.965 & 72.861 \\
\hline 2 & 0 & 107.556 & 102.625 & 107.557 & 0 \\
\hline 3 & 0 & 0 & 191.424 & 106.754 & 0 \\
\hline 4 & 0 & 0 & 278.908 & 0 & 0 \\
\hline \multicolumn{6}{|c|}{$R_{i, j}=R_{i, 0}+\Delta R_{i, j}$} \\
\hline App. Load & 73.686 & 107.556 & 278.908 & 106.754 & 72.861 \\
\hline $\operatorname{SUPP}(p)$ & 2 & 3 & 4 & 5 & 6 \\
\hline $\operatorname{STEP}(j-1)$ & 1 & 3 & 5 & 4 & 2 \\
\hline
\end{tabular}

App. Load = Load transferred to the structure after removal of support ;

*Also denoted as $\left(-R_{p, j-1}\right)$, to compute the accumulated reaction forces.

5. Numerical FE Analysis:

The following Load Cases were chosen to properly simulate the construction sequence:

Table7. List of Load Cases considered.

\begin{tabular}{|c|c|c|c|}
\hline Case & LoadType & Load & Desciption \\
\hline Text & Text & Case \# & \\
\hline $\mathrm{P}$ & Load pattern & 1 & Applied load \\
\hline TWR 2 & Load pattern & 2 & Initial React. Force SUPP.02 \\
\hline TWR 3 & Load pattern & 3 & Initial React. Force SUPP.03 \\
\hline TWR 4 & Load pattern & 4 & Initial React. Force SUPP.04 \\
\hline TWR 5 & Load pattern & 5 & Initial React. Force SUPP.05 \\
\hline TWR 6 & Load pattern & 6 & Initial React. Force SUPP.06 \\
\hline STEP 1 & Load pattern & 7 & Load distribution due to the removal of SUPP.02 \\
\hline STEP 2 & Load pattern & 8 & Load distribution due to the removal of SUPP.06 \\
\hline STEP 3 & Load pattern & 9 & Load distribution due to the removal of SUPP.03 \\
\hline STEP 4 & Load pattern & 10 & Load distribution due to the removal of SUPP.05 \\
\hline TWR 2R & Load pattern & 11 & Accumulated load = Removal of SUPP. 02 \\
\hline TWR 3R & Load pattern & 12 & Accumulated load = Removal of SUPP. 03 \\
\hline TWR 4R & Load pattern & 13 & Accumulated load = Removal of SUPP. 04 \\
\hline TWR 5R & Load pattern & 14 & Accumulated load = Removal of SUPP. 05 \\
\hline TWR 6R & Load pattern & 15 & Accumulated load = Removal of SUPP. 06 \\
\hline
\end{tabular}


These Load Cases were grouped in suitable Combinations to simulate the whole lowering operation, as shown in Table8, starting from the initial configuration (COMB. "STEP O", in which the structure is fully supported) up to the final stage (COMB. 5, which corresponds to the phase when the structure is supported only at the ends):

Table8. Load Combinations adopted to simulate the construction sequence.

\begin{tabular}{|c|c|c|c|c|c|c|c|c|}
\hline \multicolumn{9}{|c|}{ TABLE: Combination Definitions } \\
\hline \multirow{3}{*}{ Load case } & \multirow{3}{*}{$\#$} & \multicolumn{7}{|c|}{ COMB } \\
\hline & & STEP 0 & COMB 1 & СОМв 2 & COMB 3 & СомB 4 & COMB 5 & END \\
\hline & & \multicolumn{7}{|c|}{ Beggining -SUPP. 02 -SUPP. 06 -SUPP. 03 -SUPP. 05 -SUPP. 04 Final truss } \\
\hline$P$ & 1 & 1 & 1 & 1 & 1 & 1 & 1 & 1 \\
\hline TWR 2 & 2 & 1 & 1 & 1 & 1 & 1 & 1 & 0 \\
\hline TWR 3 & 3 & 1 & 1 & 1 & 1 & 1 & 1 & 0 \\
\hline TWR 4 & 4 & 1 & 1 & 1 & 1 & 1 & 1 & 0 \\
\hline TWR 5 & 5 & 1 & 1 & 1 & 1 & 1 & 1 & 0 \\
\hline TWR 6 & 6 & 1 & 1 & 1 & 1 & 1 & 1 & 0 \\
\hline STEP 1 & 7 & 0 & 1 & 1 & 1 & 1 & 1 & 0 \\
\hline STEP 2 & 8 & 0 & 0 & 1 & 1 & 1 & 1 & 0 \\
\hline STEP 3 & 9 & 0 & 0 & 0 & 1 & 1 & 1 & 0 \\
\hline STEP 4 & 10 & 0 & 0 & 0 & 0 & 1 & 1 & 0 \\
\hline TWR 2R & 11 & 0 & 1 & 1 & 1 & 1 & 1 & 0 \\
\hline TWR 3R & 12 & 0 & 0 & 0 & 1 & 1 & 1 & 0 \\
\hline TWR 4R & 13 & 0 & 0 & 0 & 0 & 0 & 1 & 0 \\
\hline TWR 5R & 14 & 0 & 0 & 0 & 0 & 1 & 1 & 0 \\
\hline TWR 6R & 15 & 0 & 0 & 1 & 1 & 1 & 1 & 0 \\
\hline
\end{tabular}

The nodal loads included in each Load Case were set according to the values previously calculated from the Influence Matrix and are presented in Table9, for illustrative purposes.

Table9. Description of loads, per Load Case

\begin{tabular}{|c|c|c|c|c|}
\hline \multicolumn{5}{|c|}{ TABLE: Joint Loads - Force } \\
\hline Joint & LoadPat & F1 & $\mathrm{F} 2$ & F3 \\
\hline Text & Text & $\mathrm{KN}$ & $\mathrm{KN}$ & $\mathrm{KN}$ \\
\hline 14 & $\mathrm{P}$ & 0 & 0 & -40.00 \\
\hline 15 & $\mathrm{P}$ & 0 & 0 & -40.00 \\
\hline 16 & $\mathrm{P}$ & 0 & 0 & -80.00 \\
\hline 19 & $\mathrm{P}$ & 0 & 0 & -80.00 \\
\hline 20 & $\mathrm{P}$ & 0 & 0 & -80.00 \\
\hline 21 & $\mathrm{P}$ & 0 & 0 & -80.00 \\
\hline 22 & $\mathrm{P}$ & 0 & 0 & -80.00 \\
\hline 3 & STEP 1 & 0 & 0 & 32.95 \\
\hline 4 & STEP 1 & 0 & 0 & 6.85 \\
\hline 5 & STEP 1 & 0 & 0 & -0.99 \\
\hline 6 & STEP 1 & 0 & 0 & -0.83 \\
\hline 3 & STEP 2 & 0 & 0 & -1.34 \\
\hline 4 & STEP 2 & 0 & 0 & 6.70 \\
\hline 5 & STEP 2 & 0 & 0 & 32.59 \\
\hline 4 & STEP 3 & 0 & 0 & 88.80 \\
\hline 5 & STEP 3 & 0 & 0 & -0.80 \\
\hline 4 & STEP 4 & 0 & 0 & 87.48 \\
\hline 2 & TWR 2 & 0 & 0 & 73.69 \\
\hline 3 & TWR 3 & 0 & 0 & 75.96 \\
\hline 4 & TWR 4 & 0 & 0 & 89.08 \\
\hline 5 & TWR 5 & 0 & 0 & 75.96 \\
\hline 6 & TWR 6 & 0 & 0 & 73.69 \\
\hline 2 & TWR 2R & 0 & 0 & -73.69 \\
\hline 3 & TWR 3R & 0 & 0 & -107.56 \\
\hline 4 & TWR 4R & 0 & 0 & -278.91 \\
\hline 5 & TWR 5R & 0 & 0 & -106.75 \\
\hline 6 & TWR 6R & 0 & 0 & -72.86 \\
\hline
\end{tabular}




\section{Lepikson Oliveira et al.}

For the listed joints of the FE model, the following node numbering applies:

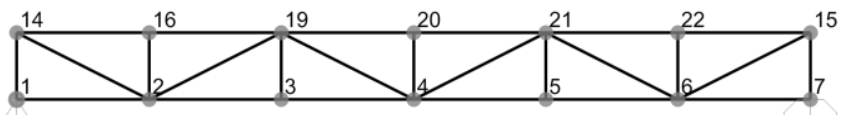

Notice that:

- Each combination contemplates the removal of a temporary support, according to the sequence previously chosen;

- To simulate the removal of a support, each Combination must comprise two different new Load Cases: one for the variation of the reaction forces on the remaining supports and the other to take into account the accumulated load applied on the structure due the removal of the tower itself;

- The Combinations were set based on the principle of superposition, so that the Combination for a later Step comprises the previous one, amended by the Load Cases corresponding to the removal of a new support and so on. Following this procedure up to the final stage, all the Load Cases are then included in the last combination;

- The Combination identified as "END" and comprising only Load Case " $P$ " was set just for verification purposes, as a simple check, since its results must be identical to those corresponding to COMB. 5.

A simple linear elastic analysis was performed in SAP 2000 and the main results are summarized below.

Comb. = STEP 0 :. Structure fully supported (Initial stage).

$\Delta \mathrm{W}_{\max }=0.089 \mathrm{~cm}$

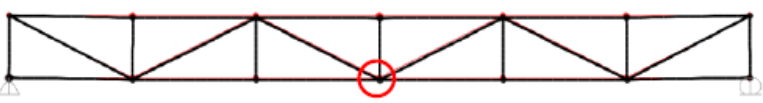

Comb. $=1:$. Removal of SUPP.02.

$\Delta \mathrm{w}_{\max }=0.231 \mathrm{~cm}$

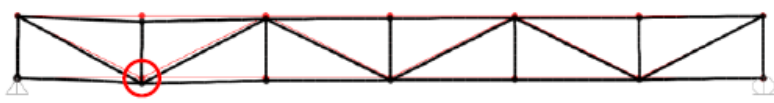

Comb. = 2 :. Removal of SUPP.06.

$\Delta \mathrm{w}_{\max }=0.229 \mathrm{~cm}$

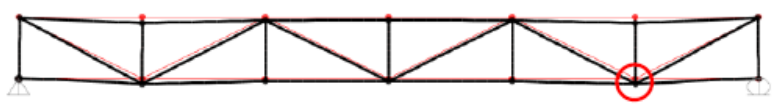

Comb. $=3$ :. Removal of SUPP.03.

$\Delta \mathrm{w}_{\max }=0.642 \mathrm{~cm}$

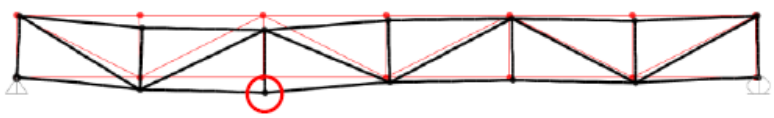

Comb. $=4$ :. Removal of SUPP.05.

$\Delta \mathrm{w}_{\max }=0.637 \mathrm{~cm}$

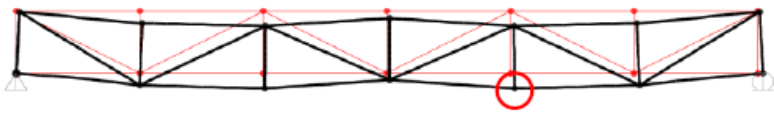

Comb. = 5 = END :. Removal of SUPP.04 (Final stage).

$\Delta \mathrm{w}_{\max }=5.839 \mathrm{~cm}$

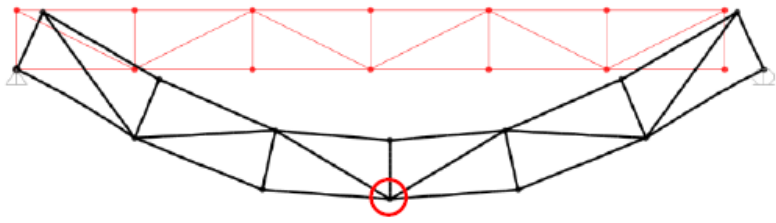

Figure7. Deformed shape of the structure during the construction sequence (displacements are multiplied by 150). 


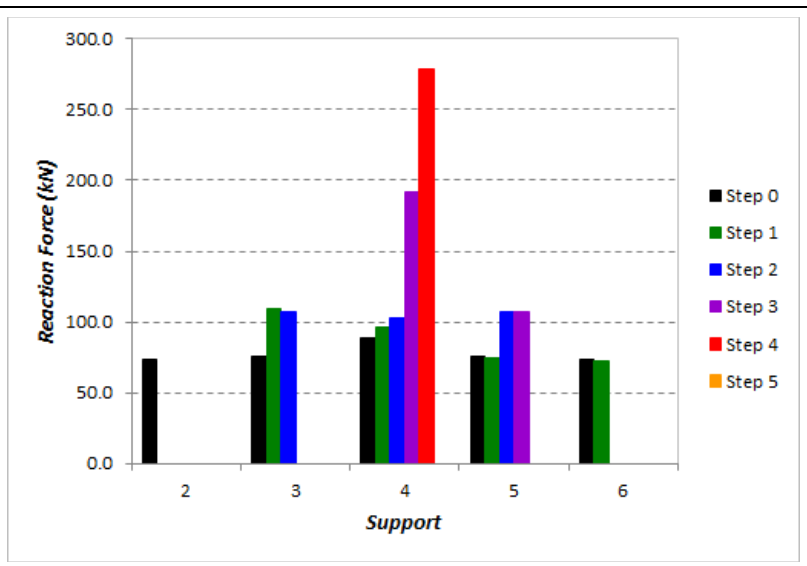

Figure8. Reaction forces, per phase (in $k N$ ).

Comb. $=$ STEP 0 :. Structure fully supported (Initial stage). $\mathrm{N}_{\max }=+13.0 \mathrm{kN}$ (BLUE); $\mathrm{N}_{\min }=-80.0 \mathrm{kN}$ (RED)

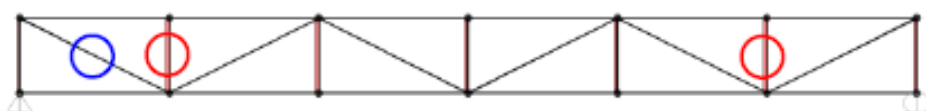

Comb. $=1$ :. Removal of SUPP.02.

$\mathrm{N}_{\max }=+94.5 \mathrm{kN}$ (BLUE); $\mathrm{N}_{\min }=-108.9 \mathrm{kN}$ (RED)

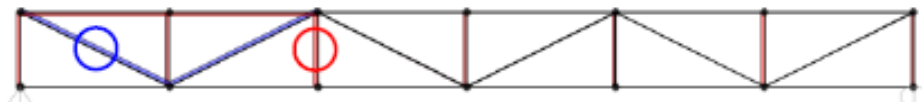

Comb. $=2$ :. Removal of SUPP.06.

$\mathrm{N}_{\max }=+91.8 \mathrm{kN}$ (BLUE); $\mathrm{N}_{\min }=-107.6 \mathrm{kN}$ (RED)

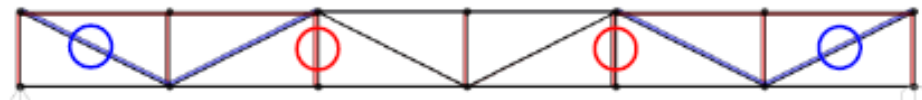

Comb. $=3$ :. Removal of SUPP.03.

$\mathrm{N}_{\max }=+153.4 k N(B L U E) ; \mathrm{N}_{\min }=-204.1 k N(\mathrm{RED})$

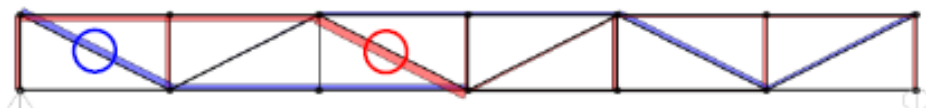

Comb. $=4$ :. Removal of SUPP.05.

$\mathrm{N}_{\max }=+135.2 \mathrm{kN}$ (BLUE) ; $\mathrm{N}_{\min }=-222.4 \mathrm{kN}$ (RED)

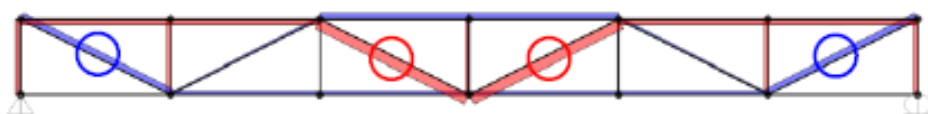

Comb. $=5$ = END :. Removal of SUPP.04 (Final stage).

$\mathrm{N}_{\max }=+640.0 \mathrm{kN}$ (BLUE); $\mathrm{N}_{\min }=-720.0 \mathrm{kN}(\mathrm{RED})$

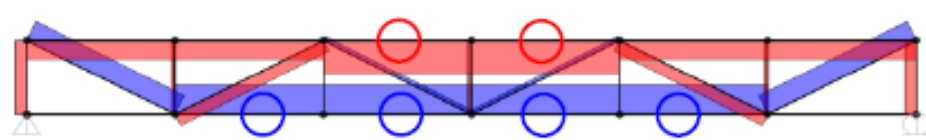

Figure9. Tension / Compression internal forces acting on the truss members, per phase (same scale for all diagrams). 
A code check was performed comprising all construction phases. The obtained results are summarized in Figure10.

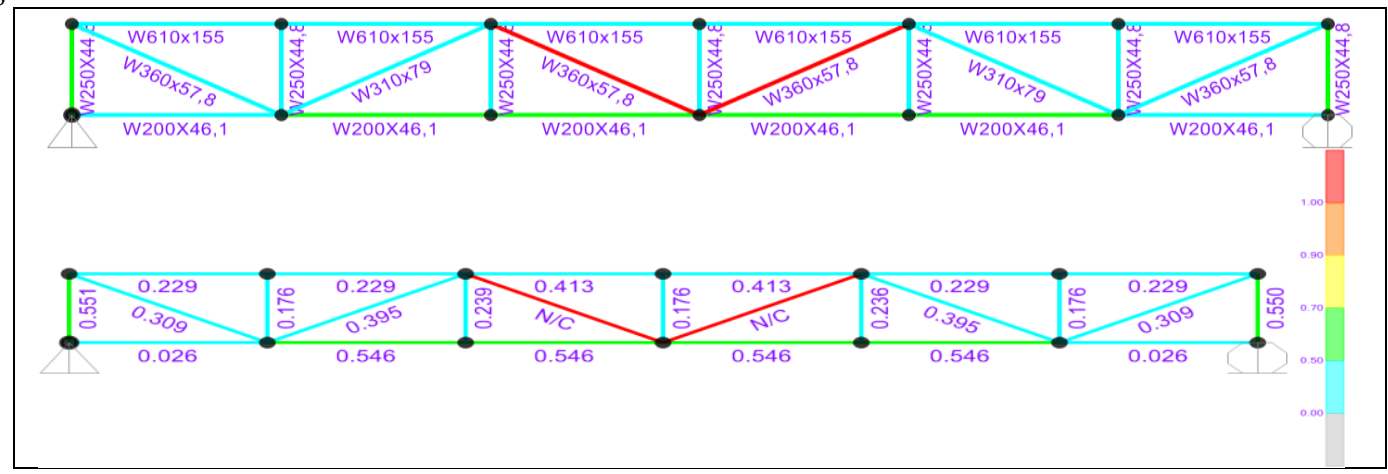

Figure10. Code check results, as per American Standard AISC/ASD (Ref. [2]).

As shown in Figure 10, two members failed the safety criteria chosen to check the structure during the construction phase. The Safety Stress Ratio(or SSR =Applied stress / Member Strength) was not automatically calculated by the post-processor available in the software for such cases, since these members are considered too slender as per the standard criteria $(\lambda>200)$. Notice that this is not an issue in the final structure, since these slender members are actually submitted to tensile forces in this configuration, so that a larger limit applies for $\lambda$ in this case $(\lambda<300)$.

Performing the code check for these members regardless of their slenderness ratio, one obtains:

$N=-204.146 \mathrm{kN}$

:.COMB.3

$f_{a}=N / A=204.146 / 72.5=2.816 \mathrm{kN} / \mathrm{cm}^{2}$

$\lambda=K \cdot l / r_{y}=(1.0 \cdot 849.43) / 3.92=228.17>200$

$F_{a}=1.988 \mathrm{kN} / \mathrm{cm}^{2}$

$\therefore$.Eq. $(E 2-2)$, Ref.[2]

$\operatorname{SSR}=f_{a} / F_{a}=1.42 \gg 1.0$

$\therefore$.Not OK!

The strength of the structural members as prescribed by Ref. [2] is presented in table 10, for illustrative purposes.

Table10. Strength of members, as per AISC/ASD

Strength of Structural Members, according to AISC/ASD 89.

$\begin{array}{lll}E= & 20103.6 \mathrm{kN} / \mathrm{cm}^{2} & F_{y}=\text { Yielding stress } \\ F_{y}= & 33.833 \mathrm{kN} / \mathrm{cm}^{2} & F_{o}=\text { Allowable stress } / \text { Compr. } \\ C_{c}= & 108.30 & F_{\mathrm{t}}=\text { Allowable stress } / \text { Tension }\end{array}$

\begin{tabular}{lrrrrrr}
\hline Profile & $\begin{array}{r}l \\
{[\mathrm{~cm}]}\end{array}$ & $\begin{array}{r}r_{y} \\
{[\mathrm{~cm}]}\end{array}$ & $\lambda$ & $\begin{array}{c}A \\
{\left[\mathrm{~cm}^{2}\right]}\end{array}\left[\mathrm{kN} / \mathrm{cm}^{2}\right]\left[\mathrm{kN} / \mathrm{cm}^{2}\right]$ \\
\hline W $610 \times 155$ & 800.0 & 7.38 & 108.40 & 198.1 & $\mathbf{8 . 8 1}$ & $\mathbf{2 0 . 3 0}$ \\
W $200 \times 46.1$ & 800.0 & 5.12 & 156.25 & 58.6 & $\mathbf{4 . 2 4}$ & $\mathbf{2 0 . 3 0}$ \\
W $250 \times 44.8$ & 400.0 & 3.50 & 114.29 & 57.6 & $\mathbf{7 . 9 3}$ & $\mathbf{2 0 . 3 0}$ \\
W $360 \times 57.8$ & 894.43 & 3.92 & 228.17 & 72.5 & $\mathbf{1 . 9 9}$ & $\mathbf{2 0 . 3 0}$ \\
W 310 $\times 79$ & 894.43 & 7.25 & 123.37 & 100.0 & $\mathbf{6 . 8 0}$ & $\mathbf{2 0 . 3 0}$ \\
\hline
\end{tabular}

\subsubsection{Relevant Commentaries and Remarks}

The main findings and conclusions obtained from the study of this simple example are summarized below:

1. As a first and general commentary, it is worth mentioning that the main calculation efforts are related to the determination of the Influence Matrix for the Reaction Forces. To accomplish this 
task, it suffices to solve a very simple structural problem, similar in complexity to a beam over an elastic foundation ${ }^{3}$, regardless of the type of the main structure to be supported by the temporary props;

2. Once the distribution of the reaction forces on the elastic supports is determined for each step, the structure is solved as if it was a statically determined problem, substituting the supports by their corresponding reactions, applied to the structure as external loads;

3. The solution of the structural problem becomes quite straightforward, simulating the whole operation by simply superposing the previously defined load cases. The displacement field and the internal forces are automatically determined for each step by means of a linear analysis, performed with the aid of a structural analysis software, which can also perform a safety check;

4. The main point to be highlighted with this example is that the integrity of the structural system is not necessarily assured during the construction phase, when the "optimized method" for the removal of the temporary supports is applied, since either the main structure or the temporary towers may not have been designed to withstand the internal forces resulting from the assembly sequence chosen by the Constructor. These structures must be checked for that specific condition beforehand. In case of either the structure, the towers or any of their members fail to fulfill the safety requirements for the chosen methodology, the Engineer may choose one of the following alternatives to overcome the problem:

- To follow the "classical approach" (or the Method of Related Deformed Shapes) in the lowering operation, releasing all the towers at the same time, but only at the end of the operation;

- To locally strengthen the structural members that fail to satisfy the safety check;

- To change the sequence to be followed in the removal of the towers, so that the structure is submitted to a "compatible" set of internal forces during the erection phase, taking into account the final loading that its members must resist, thus avoiding the need of local strengthening (which is certainly not required for the final structure, if it was correctly designed).

Option (III) above seems to be the simplest one in most cases, whenever a fast-track for dismantling the temporary towers is required.

Indeed, if one chooses the following sequence for the removal of the towers (always referring to Figure6):

\section{Supp. 04 $\rightarrow$ Supp. 02 $\rightarrow$ Supp. 06 $\rightarrow$ Supp. 03 $\rightarrow$ Supp. 05}

The structural system meets the safety requirements as prescribed by the applicable standards during the erection phase. It suffices to repeat the same steps as shown here from the very beginning, since the Influence Matrix depends on the previously chosen sequence to remove the supports. This result will not be presented here.

\section{Guidelines for a Safe Procedure to be Adopted at the Construction Site}

Having addressed and solved the problem related to the numerical simulation of the lowering operation, the focus now becomes the execution stage, aiming to achieve a safe job in the field. To do so, it is necessary to ensure that the field operation will be executed in total compliance with the design assumptions, following a certain predefined sequence exactly as considered in the performed and checked numerical simulations.

This is the main purpose to justify the elaboration of a formal procedure for the erection sequence, to be prepared by the Engineers in charge of the construction phase (but under the supervision of the designer); the matter to be briefly discussed in this section.

A formal document that resumes the "Erection Procedure" is, therefore, an essential tool to assure the achievement of a sound, safe and successful operation, which has yet the following additional purposes:

\footnotetext{
${ }^{3}$ Notice that the springs represented in Figure 5 simulate the corresponding stiffness of the temporary towers (chosen as intermediate supports).
} 
- To list and organize all the required preparation tasks to reach the specified configuration of the structural system prior to the lowering operation, as envisaged by the design;

- To gather in a sole document all the data related to a relatively complex operation, which necessarily involves a multidisciplinary team and thus promoting the compatibility and the integration of the many existing interfaces, favoring their contributions and revisions and helping to avoid potential misunderstandings (and their harmful consequences);

- To clearly define the task and the corresponding responsibility of each team, according to their expertise, as well as that of the persons responsible for the overall control of the operation;

- To present the control methodology to be adopted in monitoring the operation;

- To give an overview of the operation to be performed in the field to the mobilized personnel, so that all are aware of their obligations, their importance to the process as a whole and the possible consequences of any mistakes.

According to the professional background of the authors and their field experience in similar operations, a sound procedure should contemplate the following topics:

\subsection{Introduction}

Main purpose of the document, brief description of the structure, presentation of the erection procedure specified by the Design phase and overall presentation of the document;

\subsection{References}

A list of key documents issued by the Designer (e.g. main drawings, Technical Specifications, Calculation Reports, main assumptions and recommendations for the assembling phase, etc.);

\subsection{Engineering Data}

A brief summary of the most relevant technical information to substantiate the operation, as revealed by the performed analyses to ensure safety, such as: (a) The applicable structural requirements and checks, stating the strength limits of the main structural elements; (b) The loading assumptions (eventually comprising the definition of applicable limits for environmental loads); (c) The maximum reaction forces acting on the temporary supports (to define the capacity of the hydraulic jacks); (d) The overall displacements determined at a set of previously chosen key points on the main structure (an information required to plan and to monitor the operation); (e) Internal forces acting on a set of structural members (preferably simple Tension / Compression normal forces), at some pre-selected spots on the main structure, to be used to monitor the real response of the structural system during the operation; (f) The specifications regarding the geometrical requirements, with a clear definition of the minimum required configuration for the structural system to allow the start of the operation; the specification of the other relevant configurations related to the process, as it progresses; (g) The specifications for the execution of the preparatory tests required (e.g.in the Instrumentation installed to monitor the operation), etc. These data are used as theoretical reference values to monitor the operation, as a controlling tool.

\subsection{Concepts, Guidelines and Main Assumptions Made for the Lowering Operation}

A brief overview of the lowering operation, stating: (a) The expected theoretical behavior of the structure during this phase (from the beginning = full support up to the end =free structure); $(b)$ The breakdown of the process into a set of intermediate steps or "waves" to achieve the final displacement expected (which corresponds to the structure fully released from the construction supports); (c) The resulting number of steps / "waves", the identification of each step / "wave" (e.g. by colors: Step/Wave 1 = Green, Step/Wave $2=$ blue ... ) and the corresponding maximum vertical displacement for each step (in order to define the stroke of the jacks); $(d)$ The geometrical configuration chosen for the metal shimming system to temporarily support the structure during an intermediate step (since such interruptions are required to restore the stroke of the jacks or to temporarily suspend the operation by the end of a shift); (e) The control methodology conceived to monitor the operation, 
based on the comparison of the values measured for the controlled variables (namely: Displacements / by Topographical surveys, Reaction forces / by Hydraulic pressure, Internal forces acting on a set of previously chosen key structural members / by Instrumentation)with the corresponding reference values, obtained in the numerical simulations performed during the design phase.

\subsection{Work Teams and their Responsibilities}

This topic should contain a description of the technical team in charge of the field operation, grouped in accordance with the professional background of their components, along with their respective activities to be performed and their corresponding responsibilities, so as to ensure smooth progress and good performance in a successful and safe operation. A typical Work Team is usually composed of:(a) Hydraulic team(T.01), responsible for the installation and operation of the hydraulic devices (cylinders, hoses, gauges and pumps), to control the progressive relief on the reaction forces acting on the temporary supports; (b) Instrumentation team(T.02), responsible for the installation and operation of the electronic devices (strain gages and controlling equipment), to measure the deformation of the main structure during the operation ${ }^{4}$; (c) Land Surveying team(T.03), responsible for the surveying activities and measurements, to determine the displacements of pre-selected points on the main structure during the lowering operation, for monitoring purposes;(d) Field Workers team(T.04), to locally operate each hydraulic device and the shimming, under the supervision of the Hydraulic team, in order to keep the correct pace and synchronism during the descending movement of the jacks, thus avoiding any undesirable load distribution between the temporary supports; (e) Engineering team(T.05), responsible for the structural calculations performed during the Design phase, elaboration of the Erection Procedure and monitoring of the operation (which is based on the data collected by the other teams during the operation), defining corrective actions, based on the obtained results, if necessary; (f) Controller team (Leader)(T.06), the Engineer responsible for the coordination of the whole operation.

\subsection{Preparatory Activities Prior to the Onset of the Operation}

This item summarizes the preliminary activities to be imperatively carried out prior to the onset of the lowering operation, a handy tool for the field team responsible for its execution. These activities typically comprise: (a) Calibration of all the hydraulic device sets (Jack + Gauge + Pump) to be used in the operation (including spare components), to increase the precision in the determination of the forces through pressure measurements; (b) Installation of the calibrated hydraulic sets in their predefined positions, over the temporary towers, with traceable identification Tags; (c) Structural verification and inspection of the towers, specifying their strength and the maximum allowable load on each of them (taking also into account their support points, which may impose additional limitations); (d) Land survey measurements, to determine the initial configuration of the structural system (the starting point, for future reference);(e) Weather forecast for the planned starting date of the operation; $(f)$ Field inspection, aiming to check the compliance of the structure with the Design assumptions and the requirements of the Erection Procedure; for instance, to check if: - The structural system corresponds to the one considered in the design for the initial phase of the lowering operation; - The hydraulic system (Figure 11) and instrumentation (Figure12) were correctly installed and tested; - The required shimming is correctly installed (both to support the structure and the jacks) and the lateral guides are working properly (See Figure11); - The structural requirements (e.g. reinforcements, temporary work, assembly order, applied dead load, safety issues... ) as specified by the Designer were actually provided and the structure complies with the hypotheses assumed in the structural model; - Potential interferences may occur during the downward movement of the structure, carrying out the corresponding corrective measures, if so; $(g)$ Checklists stating all the above mentioned preparatory activities, to be chosen as a guide in the field, helping the checker to verify if these requirements were all fulfilled.

\footnotetext{
${ }^{4}$ It is worth noting that the strain gages must be installed on the surface of the structural member in a completely stress-free condition (i.e. prior to subjecting it to any load). The instrumentation must not be damaged during the installation of the member, something that is commonly a tough task in the field!
} 

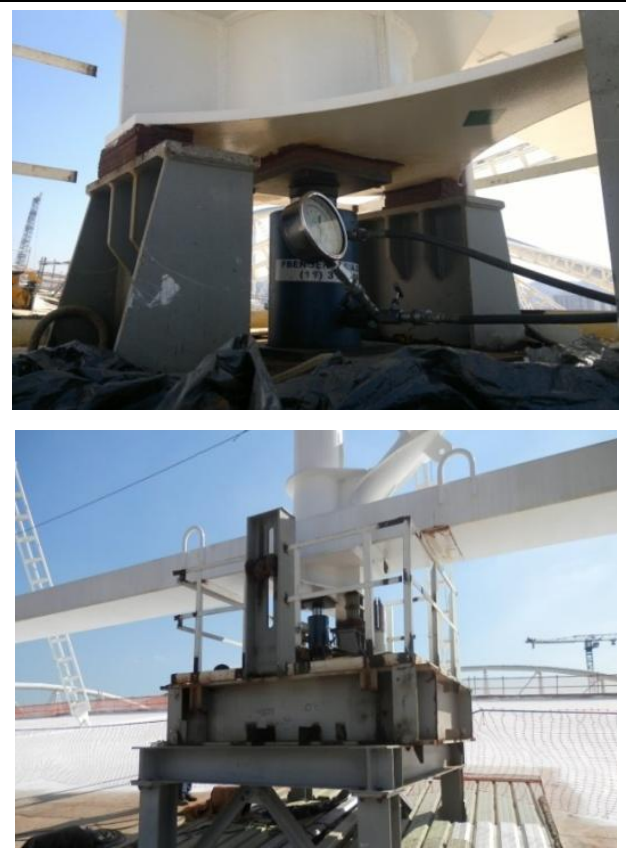

Figure11. Shimming, hydraulic devices and lateral guides installed(João Havelange Stadium, Rio de Janeiro, Brazil).

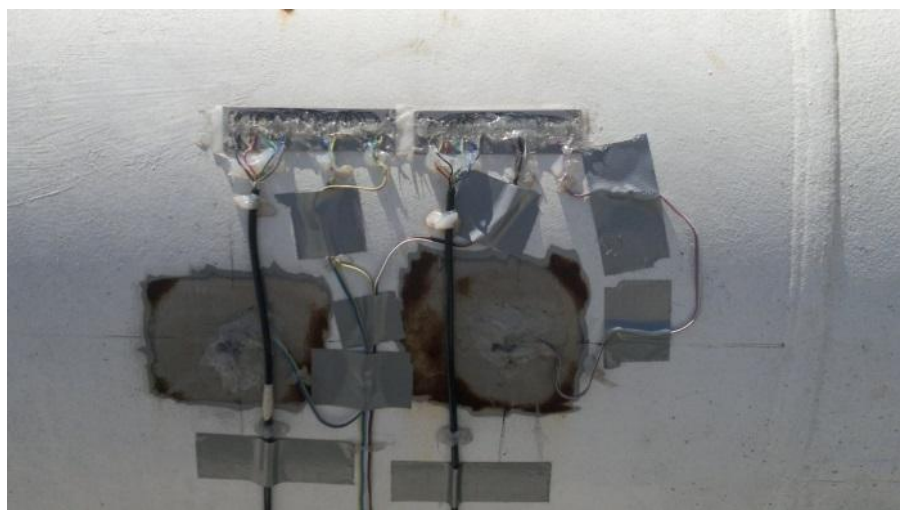

Figure12. Strain gauges installed on the surface of a key structural member, to measure the deformations as the operation progresses (João Havelange Stadium, Rio de Janeiro, Brazil).

\subsection{Full Description of the Operational Sequence}

This section summarizes all the required steps to follow in a pre-defined sequential order, whose goal is to reach the final configuration of the assembled structure (i.e. completely free from the construction supports), starting at the initial configuration, for which the main structure is still supported by the temporary towers. This is actually the core of the Erection Procedure, which should be fully described, step by step, in this topic. A typical cycle will necessarily encompass the following sequence of activities, briefly described below: (i)The Leader (T.06) authorizes the start of a new cycle $=$ Another pre-defined downward movement of the rods of the hydraulic jacks (or a controlled relief in the hydraulic pressures) to cause a corresponding relief of the reaction forces acting on the temporary towers; $\rightarrow($ ii) The Field Team(T.04) operates the hydraulic devices to obtain the target reduction of the hydraulic pressure of each jack), under the supervision of the Hydraulic Team (T.01), noting that this operation must take place simultaneously at all supports, but considering that each jack has a certain vertical displacement to fulfill; $\rightarrow$ (iii)The Leader (T.06) stops the operation temporarily and checks if the specified hydraulic pressure was actually reached on all cylinders for the current "wave", or if eventually any adjustments are required; $\rightarrow(i v)$ Once confirmed that the target pressures for the current "wave" were reached (within a certain tolerance), the Leader (T.06) informs the Engineering Team (T.05) that the step/"wave" is over and reads the measured hydraulic pressures at all supports (= Control Variables), for registration purposes. Afterwards, the Leader (T.06) authorizes the Instrumentation Team (T.02) and the Land Surveying Team (T.03) to proceed with the measurements of the deformations and displacements at the predefined control points; $\rightarrow(v)$ The 
Instrumentation Team (T.02) informs the measured deformations to the Engineering Team (T.05), for registration purposes; $\rightarrow(v i)$ The Land Survey Team (T.03) informs the measured displacements to the Engineering Team (T.05), also for registration purposes; $\rightarrow$ (vii)The Engineering Team (T.05) analyses the data obtained by the measurements in the field (= Control Variables), defines eventual corrective measures or just approves the results as such and informs the Leader (T.06) the final statement: STOP/ to perform further analyses and adjustments or GO AHEAD/ to the next step/"wave" $\rightarrow$ End of cycle.

\subsection{Control Procedures}

This section is dedicated to the description of the control procedure to be chosen in the field during the execution of the lowering operation, usually based on previously prepared EXCEL spreadsheets, containing reference (theoretical) values as determined in the design phase for the controlled variables, namely: (i) The hydraulic pressure on the jacks, corresponding to the reaction forces acting on the towers; (ii) The displacements at a set of key points on the structure and (iii) The internal forces measured at specific structural members. The reference values are determined at all steps; the real values are monitored during the operation and the measurements are compared to the corresponding reference values after each step, allowing the Engineering team (T.05) to check whether the structure is behaving as expected, or if any corrective measures are required for the next step. In this latter case, the operation must be temporarily suspended, so that the adjustments and their impacts may be determined. The spreadsheets are the main tool to guide such decisions. In any case, the Leader (T.06) is informed how to proceed: either "Proceed to the next step" or "The operation is halted for adjustments".

\subsection{List of Equipment}

This section presents a list of the equipment and devices required for the operation - Hydraulic, Instrumentation and Surveying, with their respective specifications, just for reference purposes.

\subsection{Communication System}

This section presents a suitable schematic to allow efficient communication between the teams involved in the operation, so that it may occur exactly as planned and described in Item 5.5.7. A suitable flowchart for the communication system is illustrated in Figure13.

Once the lowering operation is finished, it is recommended that a final report is issued. This report should summarize all the information collected in the field during the operation, for the controlled variables, with a comparison to the corresponding values predicted by the Design for future reference.

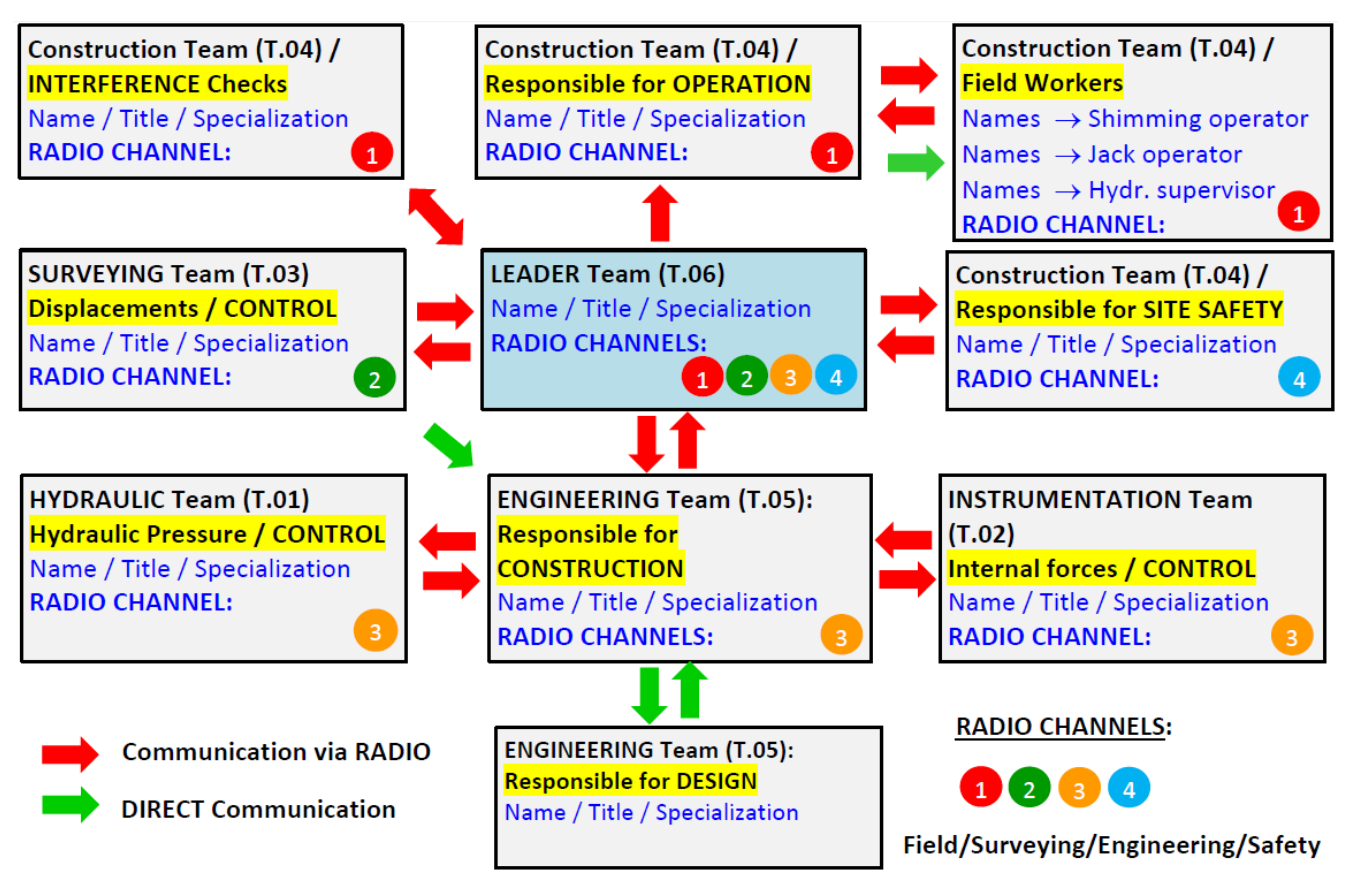

Figure13. Communication Flowchart: Description of the communication requirements to connect the teams. 


\section{Lepikson Oliveira et al.}

To end this section, it is worth presenting some valuable hints to improve the knowledge of the construction process presented here, based on the experience acquired in the execution of similar previous operations, as summarized below:

- The synchronism of the downward movements imposed by the hydraulic jacks on the structure must be assured, otherwise a migration of load between the temporary towers is prone to occur (the stiffer the structure, the more pronounced this phenomenon will be);

- One should note that there are some practical issues, which make this task even more difficult than it already is, since the displacements to impose at each supporting point are of different magnitudes (larger at mid span, smaller near the end supports), the capacity of the jacks are different (and so are their sizes), part of them are mechanically actuated and part not, etc. In order to overcome the problems generated by these issues, it suffices to consider a set of sub-steps to reach the displacement foreseen in each larger step, so that there is margin for adjustments and a pace to impose the rhythm of pumping, as the hydraulic pressure progressively increases;

- Experience has shown that the most delicate phase of the operation is the very beginning, which is the moment when the load is transferred from the shimming to the hydraulic jacks. Under these circumstances, the structure (usually very stiff) corresponds to a statically undetermined system, so the temporary supports tend to concentrate more load. A special procedure to avoid this behavior is required and shall be developed by the personnel in charge of the overall Erection Procedure;

- Lateral (horizontal) loads are not allowed to act at the top of the pistons of the hydraulic devices, which were not designed to withstand bending moments. To avoid the risk of occurrence of such actions, which can jeopardize the jacks and/or render the structural system instable while supported by the hydraulic devices, a guide system specially designed to resist such loads should be provided. These guides are fixed on the temporary towers, as illustrated in the example presented in Figure 11;

- Experience has shown that the hydraulic pressure shall be the main variable to be controlled during the operation: The pressure rules the process, the displacements of the system and the arising internal forces follow. These two last variables are measured after each step and compared to the corresponding reference values afterwards. That is the practical way to manage the operation, which also leads to a safe procedure, since the main concern is to avoid the migration of unexpected loads to a given temporary support. The hydraulic pressure is surprisingly easy to set. The authors' experience has proved that there was no difficulty to exactly impose the predetermined pressures to the jacks, simultaneously at all supports;

- Since the total vertical displacement of the structure at mid span (at the end of the lowering operation) is usually larger than the stroke of the available hydraulic jacks, a few breaks are required during the process, in order to remove a number of shim plates under the cylinders and restore the strokes. During these breaks, the structure temporarily rests on the lateral supports, upon another set of adjustable shim plates (See Figure 11). Experience has shown that it is good practice to fix the top of rods of the hydraulic jacks to the bottom side of the lifted structure. Proceeding this way, the hydraulic power itself is applied to lift up the jacks, leaving room to remove the shim plates under them, in order to adjust the height for the next step in a very quick and effective way. Note also that the use of fully extended (or fully contracted) piston rods is not recommended, or else it will no longer be possible to lift and to maneuver the structure if required (for any adjustments), whenever it is supported by the hydraulic devices;

- Since the capacity of the hydraulic cylinders is a value that varies in a discrete way, considering the equipment available in the market, it is also good practice to select the hydraulic devices with some margin to assimilate any increase in the dead load to be lifted; a value in the vicinity of 1.5 times the estimated design load is recommended. One should remember that the hydraulic devices are usually pre-calibrated to increase the precision of the pressure measurements (or, in other words, to know the error committed when the pressure is determined by $p=$ Force $\times$ Area), so that the replacement will also represent a loss of quality, besides time and money. Besides, in practice the quantities are never as estimated by the design, and the change is always on the unfavorable side; 
- Finally, it is recommended to foresee spare hydraulic devices for replacement, in case of any failures. The availability of at least one pair for each capacity is recommended, bearing in mind that the spare device sets (cylinder + gauge) shall be also pre-calibrated.

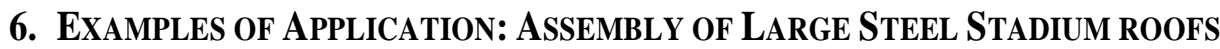

This section presents the results obtained in two operations performed by ODEBRECHT in accordance with the methodology described herein. The authors of the present work were members of the Engineering Team (T.05): at the São Paulo/ Corinthians Arena (one of the Brazilian stadia designated for the FIFA 2014 World Cup) and at the João Havelange Olympic Stadium (the one which was restored for the 2016 Olympic Games and was recently renamed as Nilton Santos Olympic Stadium, also known as Engenhão).

The following information will be presented for each one of them:

- A brief summary explaining how the structural system works;

- The measured displacements of the main structure, at a pre-selected set of key points (in $\mathrm{mm}$ );

- The measured internal forces at the main structural element(s) (in $t f$ );

- The comparison between the measured data with the corresponding reference / theoretical values.

These data were extracted from the final reports issued in the context of the Project.

\subsection{São Paulo / Corinthians Arena}

\subsubsection{Description}

The roof is composed by two huge truss-type steel structures covering the eastern and western grandstands, connected by two heavy trusses in the north and south sides, as illustrated in Figure 14. These connecting trusses are suspended by tie rods (Figure15), allowing the structure to cover a 170 $m$ long span with a cross-section only $3 \mathrm{~m}$ in height. The northern and southern trusses represent together about half the weight of the whole roof (Total Weight $=7.250 \mathrm{tf}$ ).

The structures are identical for the both the northern and southern trusses. They were assembled based on the same methodology, with modules supported by temporary towers during the erection phase, which were later removed when released by a lowering operation, executed in Dez-2104.
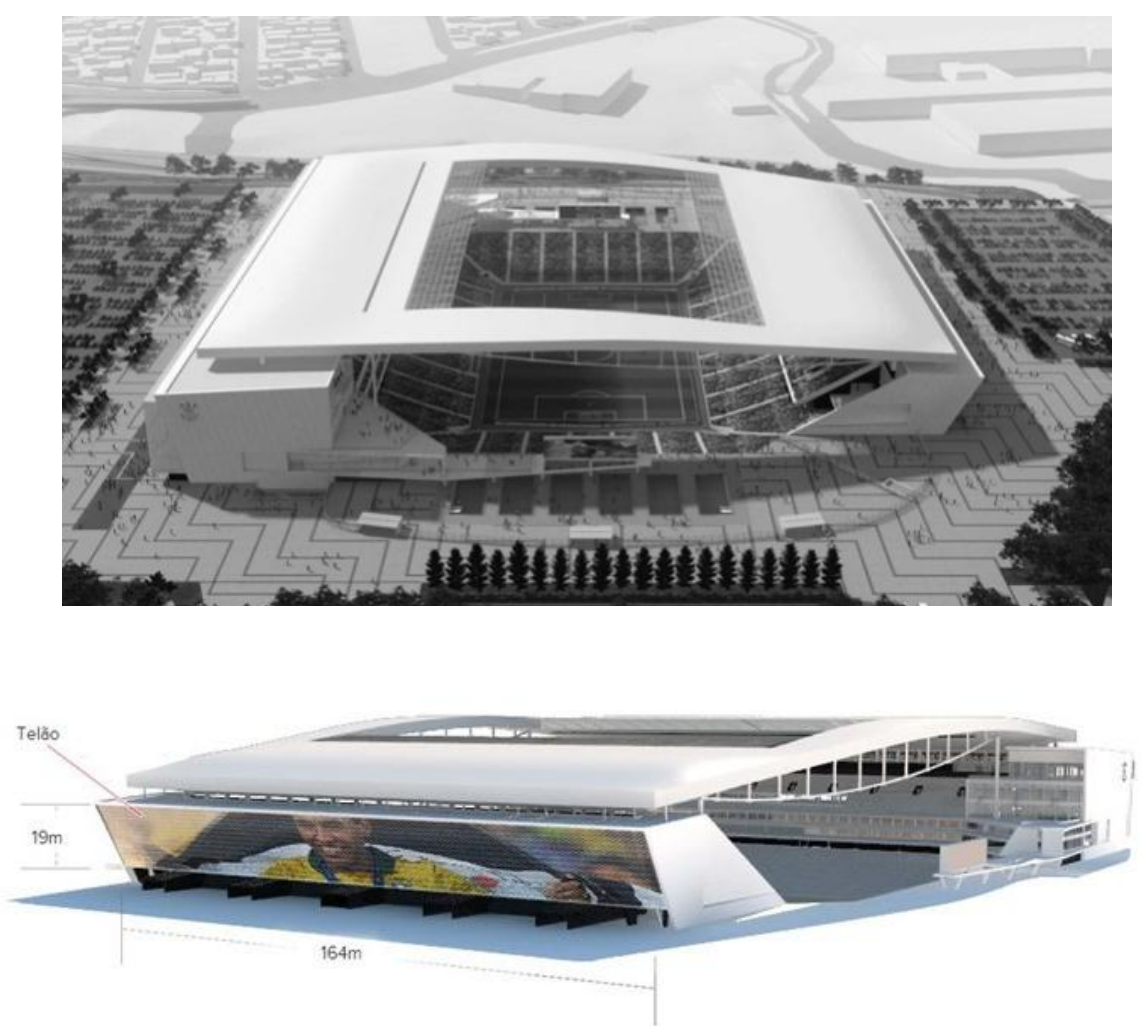

Figure14. An overview of the Corinthians Arena, Brazil. 


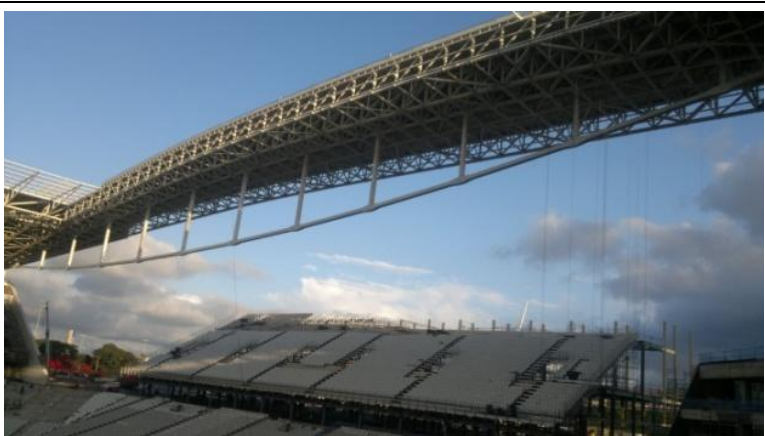

Figure15. Southern Truss of the Corinthians Arena, erection stage just finished.

The planned operation was exactly the same for both sides, but they did not occur at the same time. The southern side was released first. The data presented below refer to the southern truss (the numbers for the north are of the same order of magnitude).

\subsubsection{Support Reactions / Hydraulic Forces}

Two jacks per tower were used in the operation, with the following initial reactions (prior to the operation -- See also Figure 16): T.01 with $56 t f$ (internal) / 78tf (external); T.02 with $73 \mathrm{tf}$ (internal) / $106 t f$ (external); T.03 with 106tf (internal) / 157tf (external); T.04 with 131 tf (internal) / 171tf (external); T.05 with 177tf (internal) / 138tf (external), where internal is the side of truss facing the inside of the stadium. In Figure 16, the towers are numbered from left to the right (a similar numbering was considered for the other side).

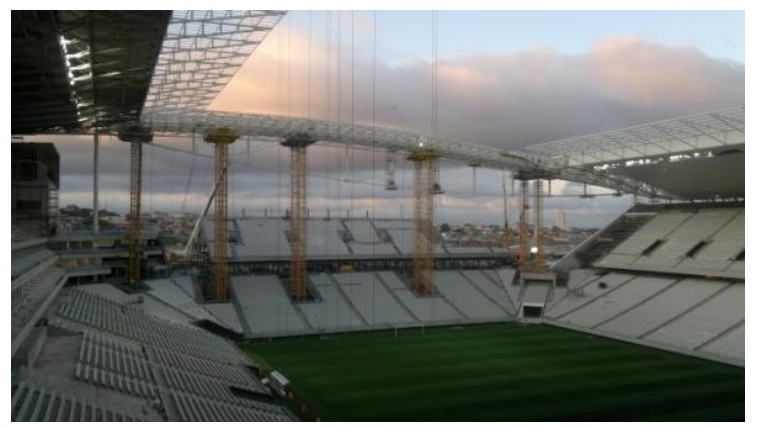

Figure16. Northern Truss of the Corinthians Arena during the erection phase.

The initially imposed pressures were: T.01 with 80bar (internal) / 110bar (external); T.02 with 115 bar (internal) / 208bar (external); T.03 with 100bar (internal) / 152bar (external); T.04 with 105 bar (internal) / 152bar (external); T.05 with 145bar (internal) / 120 bar (external). For the lifting, hydraulic jacks of 300 and 500 if capacity were used (with limit pressures of 390 and 234 bar, respectively.), distributed according to the magnitude of the provided reaction forces. The hydraulic pressure was the controlling variable during the operation, which was progressively relieved at each step (total of 6 steps), as presented in Table 11 below.

Table11. Reaction forces on each step, southern truss (in tf).

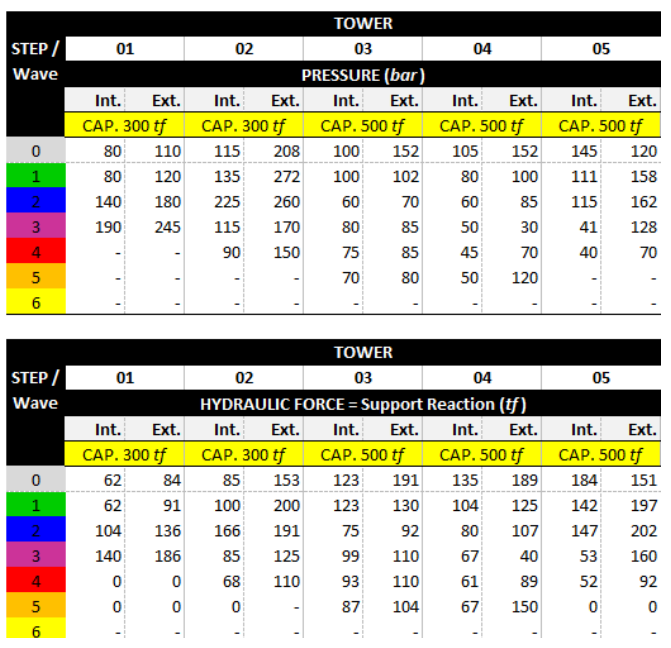


As shown in Table11, the reaction forces reached the null value only during the last steps (5 and 6), so all the towers were released almost at the same time, by the end of the operation. This is a classic example of the application of the so-called "Method of Related Deformed Shapes" approach, addressed above in Item 2 .

\subsubsection{Displacements}

The displacements were determined after each step, by Land Surveying (as prescribed in the Erection Procedure), and the measured results were compared afterwards to the corresponding reference values. The target points were located at the vertical columns, which connect the main truss to the tie rod (See Figure16). These points are also aligned to the axes of the temporary towers. The obtained displacements are graphically represented in Figure 17, as deflections in relation to the initial (almost undeformed) configuration. The curves with the theoretical / expected deflections, for each step, were also included in the same graph (with dashed lines), for comparison purposes.

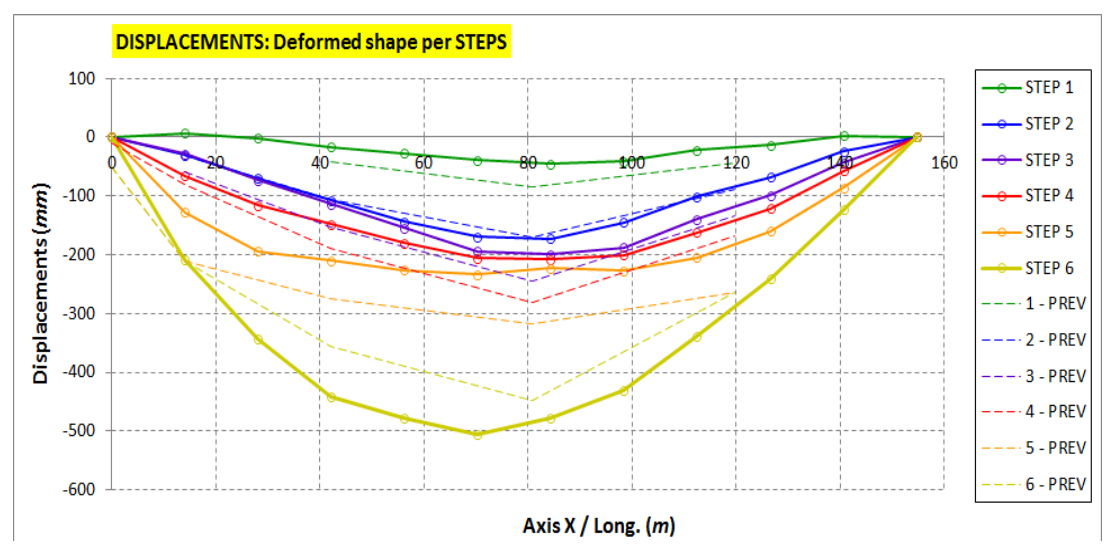

Figure17. Displacements of the southern truss, measured during the lowering operation.

\subsubsection{Internal Forces}

In this case, the following members were instrumented: the tie rod, the upper and lower chords of the truss (at mid span). In such a simple structural system, based on the theory of trusses, the tension force acting upon the rod is an excellent parameter to characterize how precise and efficient was the mathematical model to simulate the real structural behavior. The measured values for the strains were converted into the resulting tension force and the results obtained are presented in Figure18.

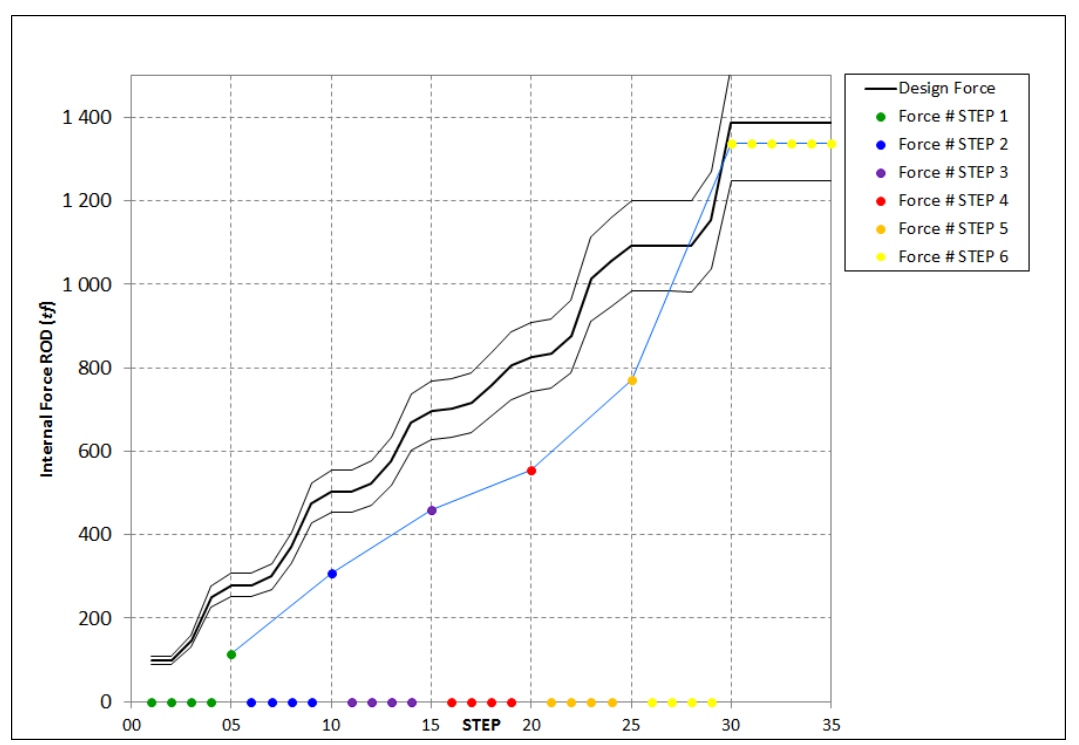

Figure18. Tension force acting upon the tie rod of the southern truss, measured during the lowering operation

It is worth noting in the data graphically presented in Figure 18, that the strains were measured only at the last sub-step of each main step during the procedure. The measured tension force reached the magnitude of $1337 \mathrm{tf}$, which represents 96\% of the theoretical value expected (Design Force $=1386$ $t f)$. Notice that the thinner black lines in the graphic represent a tolerance margin of $10 \%$ around the reference value. 
Similar results were obtained for the northern truss. The lowering operation took place in Jan-2015.

\subsection{João Havelange Olympic Stadium}

\subsubsection{Description}

The roof is composed by a ring-shaped shell structure, which is suspended by four arches. The eastern and western arches are larger; the topmost point at mid span reaches $31 \mathrm{~m}$ high (from the level of the supports, at the top of two concrete columns about $42 \mathrm{~m}$ high), covering a span $220 \mathrm{~m}$ long. The smaller northern and southern arches have the height of $23 \mathrm{~m}$ and a span $164 \mathrm{~m}$ long. The thrust of the arches are resisted by tie rods connected to their ends, so that the eight concrete columns, that support the four arches (two per corner / arch) are not submitted to significant in-plane bending moments. The suspension of the roof is composed by hangers, one pair for each main truss, reaching 20 hangers in total for the west and east sides, and 14 for the north and south. These structural members are also responsible for ensuring the stability of the arches, composing their lateral bracing system.

In its original configuration (illustrated in Figure 19), the main structural members were composed by steel pipes (arches, tie rods and hangers), whereas the shell structure in the plane of the roof is composed by steel profiles and corrugated plates in a very conventional arrangement, although not so effective. Hot-rolled steel profiles were used to compose the secondary members (main radial trusses, catwalk and the horizontal bracing system), while cold-formed slender elements were selected for the tertiary members (purlins), which are also responsible for the stability of the trusses. The total weight of the roof was $4200 \mathrm{tf}$ in this configuration (including accessories).

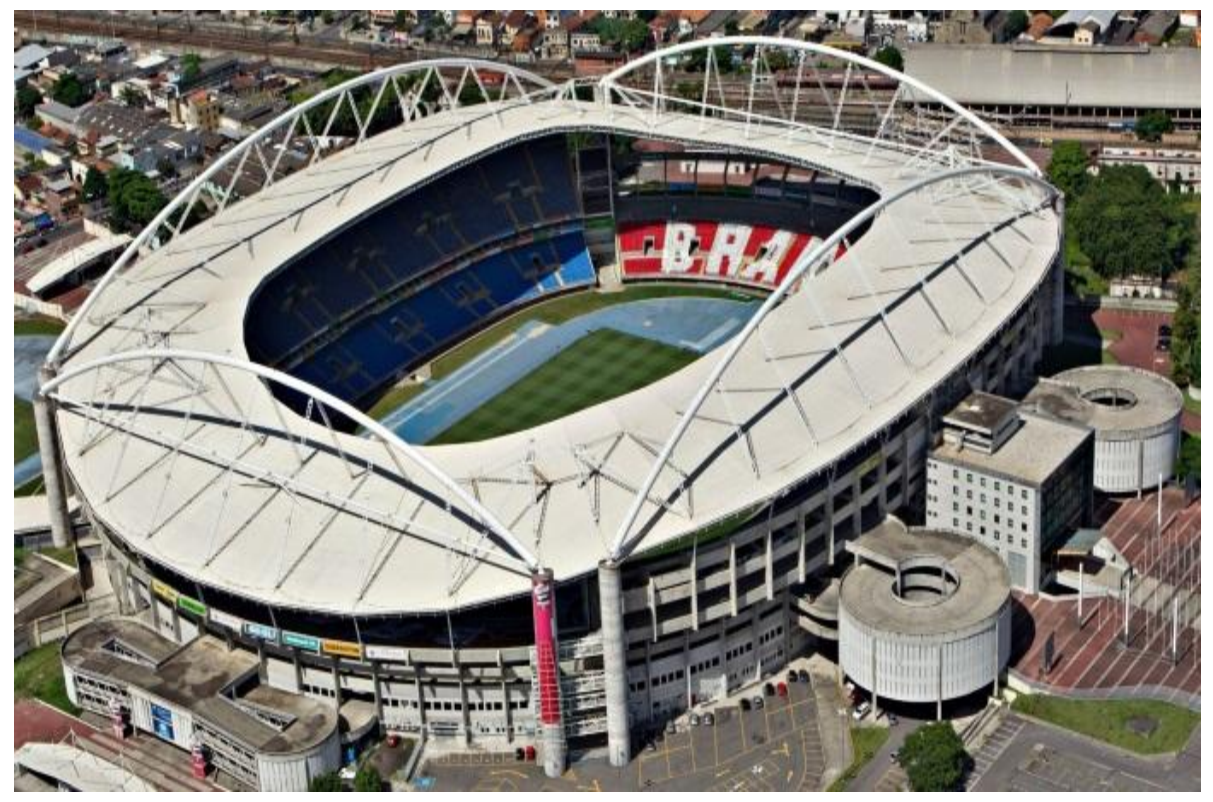

Figure19. An overview of João Havelange stadium, in its original configuration (2007), Rio de Janeiro, Brazil

The original structure was inaugurated in 2007 for the Pan-American games, but the roof presented severe structural issues ever since. The structure was the matter of extensive studies, including a series of sophisticated field measurements, as an attempt to better understand its real behavior. The causes of the structural problems, clearly a very complex and polemic matter, are not the subject of this paper and so they will no longer be discussed here. In any case, the findings of these studies led to the need of a huge reinforcement, which was executed in 2015. The main change consisted of the transformation of the arches in vertical mega-trusses, as shown in Figure20. In this picture, the overall reinforcement incorporated to the original structure is highlighted, putting in evidence the components of the mega-truss: the diagonals, vertical members and the lower chord. The intervention also comprised a general reinforcement in the structure of the roof itself (not visible in the picture), as well as a pair of cables to reinforce each concrete column. To achieve this goal, it was necessary to support the roof again, so that the reinforcement incorporated would also work for the dead load (see Figure21). The total weight of the reinforcement reached $1500 \mathrm{tf}$ (cables not included).

The final step of the construction work was the lowering operation, in order to release the structure from the previously installed (new) construction supports, which was initiated in July-2015 and represents an example of the "Influence Matrix Method" approach. 


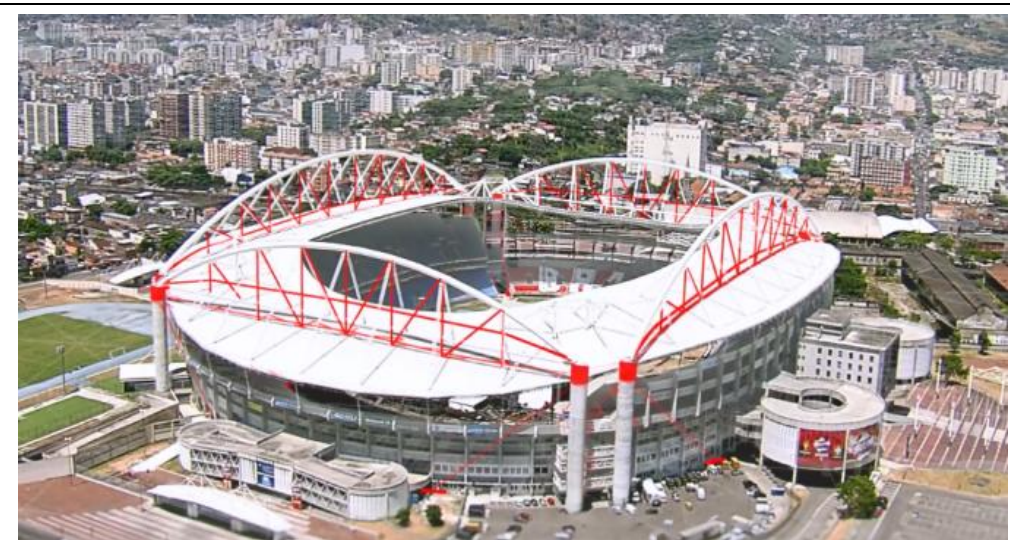

Figure20. An overview of the reinforcements installed on the roof structure of the João Havelange stadium.

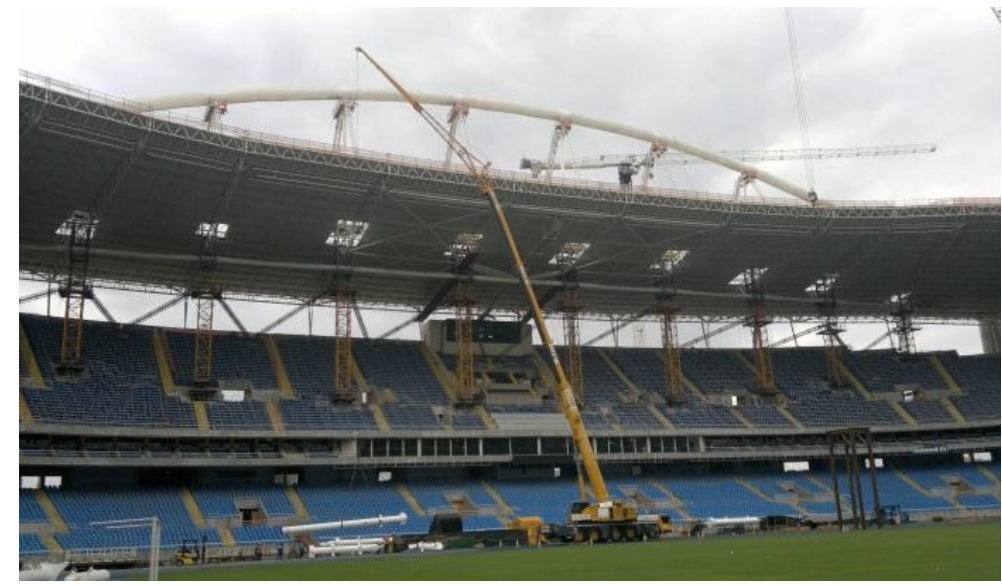

Figure21. Temporary towers to support the roof prior to the installation of the reinforcement.

The lowering operation occurred in different phases, to comply with the needs and time schedule of the construction site. The data presented in the sequence refers to the western arch.

\subsubsection{Support Reactions / Hydraulic Forces}

The number of towers (10) was determined by the number of suspension points. One jack per tower was used in the operation, with a distribution of the initial reaction forces as presented in Table12 (for "STEP 0"). In Figure21, the towers are numbered from the left to the right (according to the same numbering system considered in the design).

The hydraulic pressure was the controlling variable during the operation, which was progressively relieved at each step (6 steps in total), as presented in Table 12 below.

Table12. Reaction forces at each step, western arch (in $t f$ ).

\begin{tabular}{|c|c|c|c|c|c|c|c|c|c|c|}
\hline \multirow{5}{*}{$\begin{array}{l}\text { STEP / } \\
\text { Wave }\end{array}$} & \multicolumn{10}{|c|}{ TOWER } \\
\hline & 01 & & \multicolumn{2}{|c|}{02} & \multicolumn{2}{|c|}{03} & \multicolumn{2}{|c|}{04} & \multicolumn{2}{|c|}{05} \\
\hline & \multicolumn{10}{|c|}{ PRESSURE (bar) } \\
\hline & \multicolumn{10}{|c|}{ Cylinder CAPACITY ( $t f)$} \\
\hline & 200 & 200 & 200 & 200 & 200 & 300 & 200 & 200 & 300 & 145 \\
\hline 0 & 245 & 260 & 430 & 470 & 415 & 258 & 465 & 500 & 290 & 250 \\
\hline 1 & 31 & 168 & 288 & 358 & 352 & 219 & 356 & 372 & 195 & 72 \\
\hline 2 & - & 134 & 230 & 286 & 281 & 175 & 285 & 297 & 156 & - \\
\hline 3 & - & - & 115 & 215 & 211 & 131 & 214 & 149 & - & - \\
\hline 4 & - & - & - & 143 & 141 & 88 & 142 & - & - & - \\
\hline 5 & - & - & - & 72 & 70 & 44 & 71 & - & - & - \\
\hline \multirow[t]{2}{*}{6} & - & - & - & - & - & - & - & - & - & - \\
\hline & \multicolumn{10}{|c|}{ AXIS } \\
\hline STEP / & 31 & 33 & 35 & 37 & 39 & 41 & 43 & 45 & 47 & 49 \\
\hline \multirow[t]{3}{*}{ Wave } & \multicolumn{10}{|c|}{ HYDRAULIC FORCE = Support Reaction (tf ) } \\
\hline & \multicolumn{10}{|c|}{ Cylinder CAPACITY (tf) } \\
\hline & 200 & 200 & 200 & 200 & 200 & 300 & 200 & 200 & 300 & 145 \\
\hline 0 & 71 & 76 & 125 & 137 & 121 & 120 & 135 & 145 & 135 & 53 \\
\hline 1 & 9 & 49 & 84 & 104 & 102 & 102 & 104 & 108 & 91 & 15 \\
\hline 2 & - & 39 & 67 & 83 & 82 & 82 & 83 & 86 & 73 & - \\
\hline 3 & - & - & 34 & 62 & 62 & 61 & 62 & 43 & - & - \\
\hline 4 & - & - & - & 42 & 41 & 41 & 41 & - & - & - \\
\hline 5 & - & - & - & 21 & 21 & 20 & 21 & - & . & - \\
\hline 6 & - & - & - & - & - & - & - & - & - & - \\
\hline
\end{tabular}




\section{Lepikson Oliveira et al.}

\subsubsection{Displacements}

The displacements were determined after each step, by Land Surveying (as prescribed in the Erection Procedure), and the measured results were compared afterwards to the corresponding reference values. The target points were located at the top of the arch, one for each axis (and so aligned to the towers). The obtained displacements are graphically represented in Figure22, as deflections in relation to the initial configuration (almost undeformed and relieved). The curves with the theoretical / expected deflections for each step were also included in the same graph (with dashed lines), for comparison purposes.
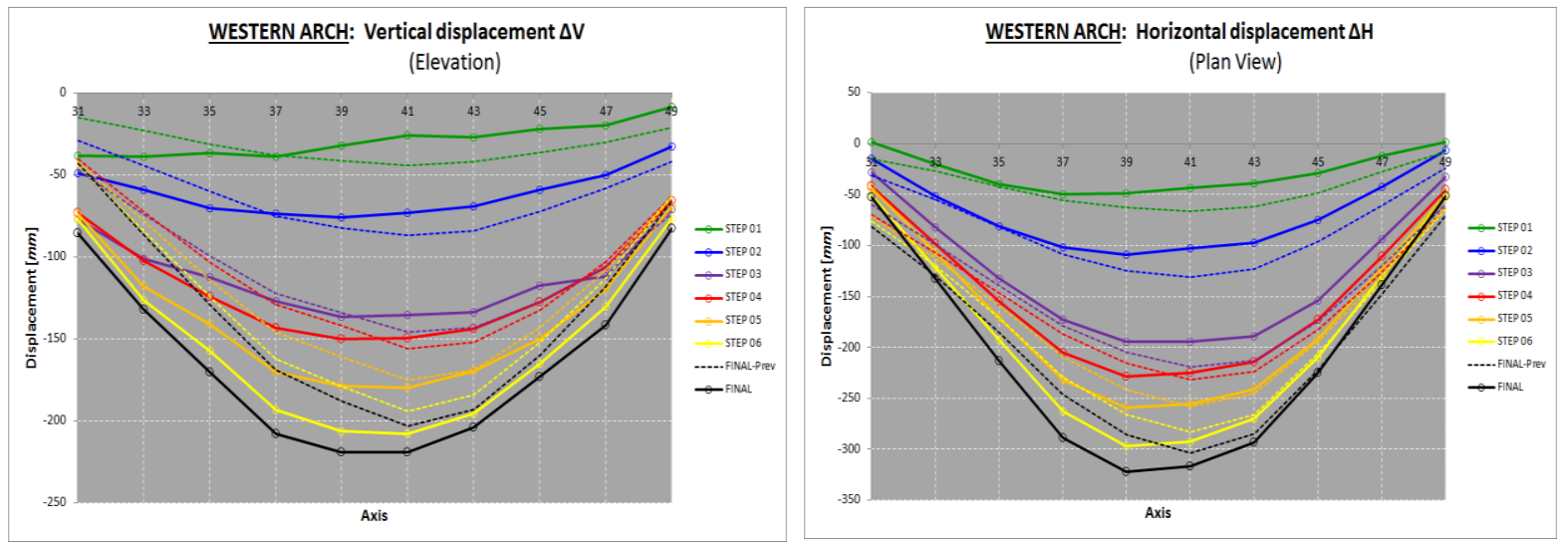

Figure22. Displacements of the western arch, measured during the lowering operation.

\subsubsection{Internal Forces}

In this case, the following members were instrumented: the arch (near the ends, where the internal forces are larger, but away from the introduction of the load at the pad eye) and the tie rod (at mid span, being the normal force almost uniform). The new structural system is still very simple, to render the measured internal forces as quite good and straightforward parameters to qualify how precise and efficient was the mathematical model to simulate the real structural behavior. The measured values of the strains were converted into the resulting tension force and the obtained results are presented in Figure23.

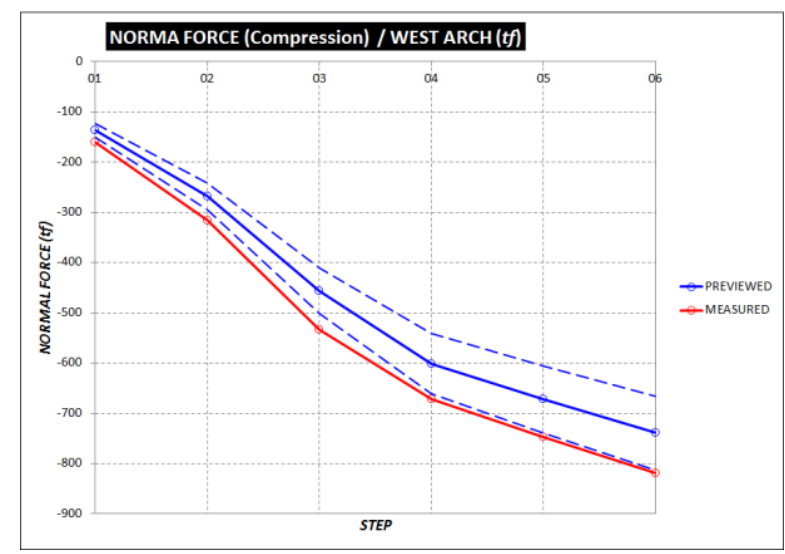

A

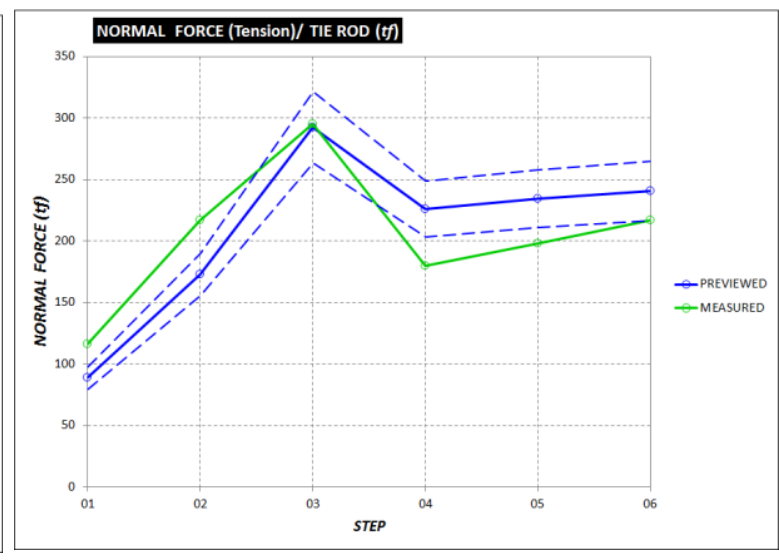

B

(A) Compression forces acting upon the arch. (B)Tension forces acting upon the tie rod.

Figure23. Forces acting on the arch (under compression) and the tie rod (under tension) on the west side, measured during the lowering operation.

In Figure23, the dashed lines represent a margin of $10 \%$ around the theoretical (previewed) value, proving that the structure behaved pretty much as expected, in accordance with to the numerical models adopted in the simulation of the operation.

The remarkable break in the line representing the internal tension force on the tie rod occurred as a result of the planned construction sequence. Indeed, it corresponds to the relief in the normal force acting upon the rod due to the installation and tensioning of the pair of cables required to reinforce the concrete columns. 


\section{Final REMARKS}

This article was dedicated to the study of the Engineering activities involved in the task of safely releasing a newly-assembled structure from the set of temporary supports used during the construction phase, referred to herein as a lowering operation. This study addresses both the design phase, presenting two approaches for the numerical model used in the simulation of the operation; as the execution phase, highlighting some important points and details that one should consider in the elaboration of a field procedure for the implementation of the operation at the construction site.

A lowering operation is simply a safe method to progressively load the structure with its self-weight, aiming to achieve the final configuration of the erection phase, by controlling the reaction forces acting upon the temporary supports with hydraulic devices, in order to:

- Avoid the migration of loading to any of the temporary supports, which could jeopardize their structure (usually steel towers);

- Avoid the premature loss of any temporary support in the beginning of the process, which could lead to the situation mentioned above and increase the risk of the operation;

- Ensure that the application of the dead load is performed in a very smooth and slow way, in order to avoid any impact and resulting dynamic effects.

Based on the statements listed above, it becomes clear that the most delicate stage of this process is the beginning of the operation, when the magnitudes of reaction forces are highest. Indeed, this stage also comprises the installation of the hydraulic devices, which requires first the lifting of the structure by means of hydraulic power, in order to transfer the support points from the shimming plates (over which the modules were initially supported, during the assembling stage) to the jacks. This is actually a very tricky and sensitive operation, that demands a totally dedicated procedure prior to its execution, given the possibility of the loading distribution in a statically undetermined structure (as is always the case), also including in this case the perturbation arising from the thermal loading.

The main differences revealed by the comparison of the two different approaches, here presented, may be summarized as follows:

- If the "Method of related deformed shapes" is followed, there is no need to check the structure for the transitory phases, during the erection sequence, since the most severe stages, to which the structure is submitted, are necessarily the first and the last ${ }^{5}$ (i.e. the initial continuous structure; and the final structure submitted to dead load - this last configuration is never the most severe one, since the structure is supposed to bear also variable loads);

- On the other hand, if the "Influence matrix method for the reaction forces" is chosen (as an attempt to optimize the removal of the temporary towers), it will be indeed necessary to check the erection sequence, in order to avoid the overstressing of any member of the structure during these transient stages. If any problem arises during the erection sequence, the solution is not necessarily a reinforcement (which is always a big issue in such situations), but the choice of a more suitable sequence to remove the towers, as shown in the simple example here presented.

As a practical tip, it is strongly recommended for the Engineering team responsible for the operation to be very judicious in the definition of the set of points to collect the data to be used in the control activities, especially for the instrumentation devices. This is a general recommendation which is particularly true and valuable in this case. A huge amount of data will be more disturbing than helpful as a tool to understand the real behavior of the structure. In this case, the less, the better (supposing, of course, a well-chosen set of key points, on members preferably submitted to a uniform stress state).

Finally, it is worth mentioning that, when correctly performed, the lowering operation is just a procedure to safely reach a configuration, in which the newly-assembled structure is totally free from

\footnotetext{
${ }^{5}$ The structural checks for the lifting of the modules, during the assembling stage were not mentioned nor considered here.
} 
the temporary supports and becomes submitted to the dead load. Therefore, it has no effect upon the final behavior of the structure, which obviously depends only on the design originally conceived (assuming a sound erection procedure). In case of any problem of this nature, the Engineering team will be aware of this fact in advance, as a result of the controlling procedure, so that the operation may be safely suspended. Corrective measures may be planned in advance and promptly implemented, in order to minimize, as much as possible, potential losses.

\section{ACKNOWLEDGMENTS}

The authors would like to sincerely thank the members of the other teams (Hydraulics, Instrumentation, Land Surveying and Construction), which suggested many amendments for the erection procedure initially proposed; these suggestions and improvements significantly contributed for the success of the operations presented here.

The authors would also like to express sincere acknowledgments to CONSTRUTORA NORBERTO ODEBRECHT, responsible for the construction of the stadia here presented, which gave us full support and placed great confidence in our work, thus enabling the fulfillment of these difficult tasks within the deadlines imposed by the realization of important events of global reach.

\section{REFERENCES}

[1] Computer \& Structures: SAP 2000 Plus, Structural Analyses Program, Version 17.3.0, 2015;

[2] American Institute of Steel Construction (AISC): Manual of Steel Construction, Allowable Stress Design, $9^{\text {th }}$ ed., 1989.

\section{AUTHORS' BIOGRAPHY}

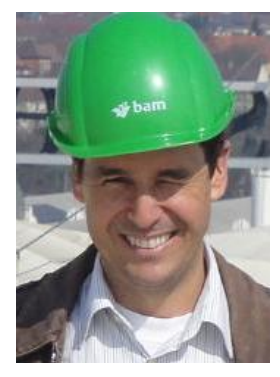

D. Lepikson Oliveira

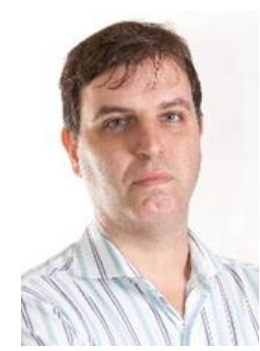

F. Rubin

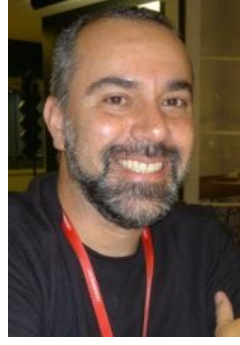

M. B. Pinheiro

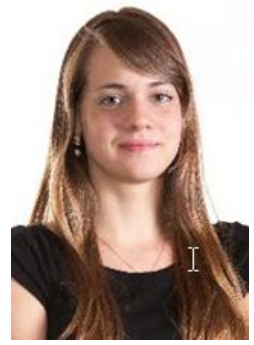

D. C. Braga

Daniel Lepikson Oliveira, Flavio Rubin, Marcelo Brisola Pinheiro and Debora Coting Braga, are part of the Structural Engineers Team of ODEBRECHT Engenharia Industrial, the industrial branch of the Construtora Norberto Odebrecht (CNO), the largest construction company in Brazil. They work in the Construction Methods Division, which aims to provide qualified technical support and Engineering background for different segments of the ODEBRECHT group.

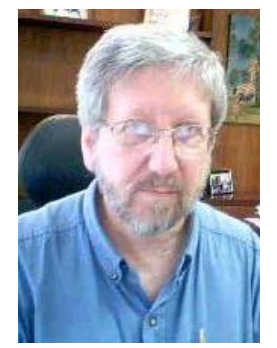

N. Szilard Galgoul

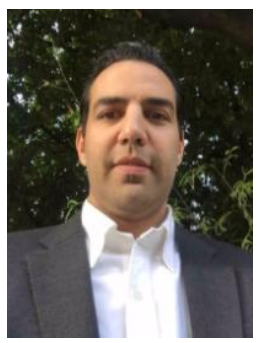

A. R. Sarabi

Professor Nelson Szilard Galgoul(*) and Eng. Aidin R. Sarabi, are consultants of NSG Engenharia Ltda., a company specialized in Offshore Engineering that also has a large experience with Civil Structures. NSG is an ODEBRECHT's recurring partner in providing technical assistance when it deals with complex structures or special projects.

(*) Federal Fluminense University, Rio de Janeiro, Brazil. 


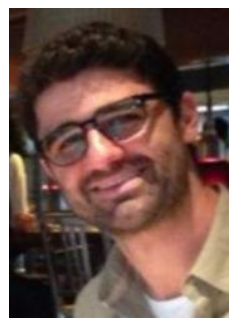

L.F.S. Macedo

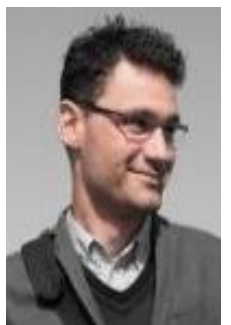

R. S. Macedo

Luiz F. Scudelari de Macedo and Rafael Scudelari de Macedo, are consultants of EMASA Engenharia Ltda., a company specialized in Structural Engineering, Design of Steel Structures, Constructability and Heavy Lifting. EMASA is also an ODEBRECHT's customary partner and it has worked in cooperation with the Construction Methods Division in many projects.

Citation: Oliveira, D. and Galgoul, N. (2017). Lowering Operations of Large Steel Structures: Numerical Simulation \& Guidelines for a Safe Procedure. International Journal of Constructive Research in Civil Engineering, 3(2), pp.1-33.

Copyright: (ㅇ 2017 Oliveira, D, et al. This is an open-access article distributed under the terms of the Creative Commons Attribution License, which permits unrestricted use, distribution, and reproduction in any medium, provided the original author and source are credited 